\title{
Existence and stability of stationary solution to compressible Navier- Stokes-Poisson equations in half line
}

\author{
Lei Wang, Guojing Zhang and Kaijun Zhang * \\ School of Mathematics and Statistics, Northeast Normal University, \\ Changchun 130024, P.R.China
}

\begin{abstract}
In this paper, we investigate the asymptotic stability of the stationary solution to the outflow problem for the compressible Navier-Stokes-Poisson system in a half line. We show the existence of the stationary solution with the aid of the stable manifold theory. The time asymptotic stability of the stationary solution is obtained by the elementary energy method. Furthermore, for the supersonic flow at spatial infinity, we also obtain an algebraic and an exponential decay rate, when the initial perturbation belongs to the corresponding weighted Sobolev space. The proof is based on a time and space weighted energy method.

Keywords. Compressible Navier-Stokes-Poisson equation, outflow problem, boundary layer, asymptotic stability, convergence rate, weighted energy estimates.
\end{abstract}

2010 Mathematics Subject Classification. 35B35, 35B40, 76N15.

\section{Introduction}

The compressible Navier-Stokes-Poisson (called NSP in the sequel for simplicity) system may be used to simulate the transport of charged particles under the influence of electrostatic force governed by the self-consistent Poisson equation (cf. [2]). In this paper, we consider the following NSP equations in the half line $\mathbb{R}_{+}:=(0, \infty)$ :

$$
\left\{\begin{array}{l}
\rho_{t}+(\rho u)_{x}=0 \\
(\rho u)_{t}+\left(\rho u^{2}+p(\rho)\right)_{x}=\mu u_{x x}+\rho E \\
E_{x}=\rho-\rho_{+}
\end{array}\right.
$$

* : The corresponding author

E-mail address: wanglei345@126.com(L. Wang); zhangkj201@nenu.edu.cn(K. Zhang)

(C) 2016. This manuscript version is made available under the Elsevier user license http://www.elsevier.com/open-access/userlicense/1.0/ 
together with the initial conditions

$$
\begin{gathered}
(\rho, u)(0, x)=\left(\rho_{0}, u_{0}\right)(x), \lim _{x \rightarrow \infty}\left(\rho_{0}, u_{0}\right)(x)=\left(\rho_{+}, u_{+}\right), \\
\inf _{x \in \mathbb{R}_{+}} \rho_{0}(x)>0, \rho_{+}>0
\end{gathered}
$$

and the boundary conditions

$$
\left\{\begin{array}{l}
u(t, 0)=u_{b}<0, \\
E(t, \infty)=0
\end{array}\right.
$$

Here the unknown functions are the density $\rho$, the velocity $u$ and the electric field $E$. The pressure $p$ is assumed to be a function depending only on the density given by

$$
p=p(\rho)=K \rho^{\gamma}
$$

for $K>0$ and $\gamma \geq 1$. In the equations (1.1), the positive constants $\mu$ and $\rho_{+}$represent the viscosity coefficient and the doping profile, respectively. In addition, $u_{+}$and $u_{b}$ are also constants. Further we suppose that the compatibility conditions on $u(t, x)$ of order 0 and 1 hold at the origin $(0,0)$. It is worth noticing that from the electric field equation $(1.1 \mathrm{c})$ and the boundary data (1.3b), the initial value $E_{0}:=E(0, x)$ can be determined by the initial density $\rho_{0}$. The condition (1.3a) implies that the fluid blows out through the boundary $\{x=0\}$. This is the reason why the problem (1.1)-(1.3) is called the outflow problem in some literature. In this case, the characteristic speed of the hyperbolic equation (1.1a) for the density $\rho$ is negative around the boundary $\{x=0\}$, so we do not need the assumption of $\rho(t, 0)=\rho_{b}$ for the well-posedness.

Recently, some important progress was made for the compressible NSP system. The global existence and the optimal time convergence rates of the classical solution around a constant state were obtained in $[7,13,12,25]$. The global strong solution to the one-dimensional non-isentropic NSP system with large data for density-dependent viscosity was established by Tan-Yang-ZhaoZou [21]. The pointwise estimate of the solution was discussed in [23]. The global well-posedness in the Besov type space for the NSP system was also proved in [6]. From the above work, it is not difficult to find the elementary fact that the momentum of the NSP system decays at the slower rate than that of the compressible Navier-Stokes system in the absence of the electric field. This fully demonstrates that the electric field could affect the large time behavior of the solution. In addition, the quasi-neutral limit and some related asymptotic limits were considered in $[4,22]$, and the global existence and nonexistence were discussed in $[3,1]$.

In general, the large-time behavior of solutions to (1.1) in the half space is much more complicated than that for the Cauchy problem, cf. [8, 11, 15, 16, 17] and references therein. For the single quasineutral Navier-Stokes system (1.1a) and (1.1b) with $E=0$, Kawashima, Nishibata and Zhu [10] proved the existence and the asymptotic stability of the stationary solution for the outflow problem. The convergence rate for this stability result was obtained by Nakamura, Nishibata and Yuge in [18], if the initial perturbation decays in a spatial direction. Furthermore, when the doping profile is zero, Duan-Yang [5] proved the stability of rarefaction 
wave and boundary layer for the outflow problem on the two-fluid NSP equations. Zhou-Li [26] studied the convergence rate of corresponding solutions toward the same boundary layer. As a continuation of the above study, the generalization of this one-dimensional outflow problem to the multi-dimensional half space problem was studied by Kagei, Kawashima, Nakamura and Nishibata in $[19,9]$.

The main concern of the present paper is to extend these results to the compressible NSP system with the nonzero doping profile. Specifically, we find a new stationary solution called the boundary layer and show the asymptotic stability of the stationary solution as well as the convergence rate for the outflow problem (1.1)-(1.3). We focus on the influence of the electric field on the existence and the stability of the stationary solution. Compared to the isentropic model in [10], our problem is more general and more complex for the electric field is taken into account. For instance, in order to obtain the existence of stationary solution, we have to introduce the new variable to deduce the stationary equations to a $3 \times 3$ system of autonomous ordinary differential equations, and examine dynamics around an equilibrium by applying the manifold theory. What's more, as we mentioned before, the slower time decay rate of the system caused by the appearance of the electric field produces some additional difficulties of analysis. Precisely, if $\chi$ denotes the perturbation of the electric field and $\psi$ denotes the perturbation of the velocity, then $\chi$ and $\psi$ may not be expected to be time space integrable. Thus, when we discuss the asymptotic stability of a nontrivial profile $(\tilde{\rho}, \tilde{u}, \tilde{E})$, it is impossible to use $\int_{0}^{\infty} \int_{0}^{\infty}|\chi(\tau, x)|^{2} d x d \tau$ and $\int_{0}^{\infty} \int_{0}^{\infty}|\psi(\tau, x)|^{2} d x d \tau$ to control the trouble terms $\int_{0}^{\infty} \int_{0}^{\infty}|\tilde{u}(x)||\chi(\tau, x)|^{2} d x d \tau$ and $\int_{0}^{\infty} \int_{0}^{\infty}|\tilde{E}(x)||\varphi(\tau, x) \psi(\tau, x)| d x d \tau$ in the basic energy estimates, respectively. Notice that $\tilde{u}$ and $\tilde{E}$ have very good space decay properties. Hence, we can establish the Poincaré type inequality, and successfully deal with the above difficulty by means of this inequality.

Notations. Throughout this paper, $H^{s}\left(\mathbb{R}_{+}\right)$denotes the general Sobolev space over $\mathbb{R}_{+}$in the $L^{2}$ sense with norm $\|\cdot\|_{s}$. For the sake of simplicity, we also use $\|\cdot\|$ for $\|\cdot\|_{0}$ without any ambiguity. We denote by $C\left([0, \infty), H^{s}\left(\mathbb{R}_{+}\right)\right)$the space of continuously functions on the interval $[0, \infty)$ with values in $H^{s}\left(\mathbb{R}_{+}\right) \cdot L^{2}\left([0, \infty), H^{s}\left(\mathbb{R}_{+}\right)\right)$is the space of $L^{2}$-functions on $[0, \infty)$ with values in $H^{s}\left(\mathbb{R}_{+}\right)$. Let $\alpha, \beta \in(0,1), k$ and $l$ stand for nonnegative integers. $\mathcal{B}^{\alpha}\left(\mathbb{R}_{+}\right)$denotes the space of the Hölder continuous functions over $\mathbb{R}_{+}$with the Hölder exponent $\alpha$ with respect to $x$. $\mathcal{B}^{k+\alpha}\left(\mathbb{R}_{+}\right)$denotes the space of functions satisfying $\partial_{x}^{i} u \in \mathcal{B}^{\alpha}\left(\mathbb{R}_{+}\right)$equipped with the norm $|\cdot|_{\mathcal{B}^{k+\alpha}}$, for $i=0, \ldots, k$. For $T>0, \mathcal{B}^{\alpha, \beta}\left([0, T] \times \mathbb{R}_{+}\right)$denotes the space of the Hölder continuous functions over $[0, T] \times \mathbb{R}_{+}$with the Hölder exponents $\alpha$ and $\beta$ and with respect to $t$ and $x$, respectively. $\mathcal{B}^{k+\alpha, l+\beta}\left([0, T] \times \mathbb{R}_{+}\right)$denotes the space of functions satisfying $\partial_{t}^{i} u, \partial_{x}^{j} u \in \mathcal{B}^{\alpha, \beta}\left([0, T] \times \mathbb{R}_{+}\right)$ equipped with the norm $|\cdot|_{\mathcal{B}_{T}^{k+\alpha, l+\beta}}$, for $i=0, \ldots, k$ and $j=0, \ldots, l$. Throughout this paper, $C$ and $c$ stand for some general positive constants. $C(T)$ is a positive constant depending on $T,\left|\varphi_{0}\right|_{\mathcal{B}^{1+\sigma}},\left|\psi_{0}\right|_{\mathcal{B}^{2+\sigma}},\left\|\left(\varphi_{0}, \psi_{0}\right)\right\|_{1}$ and $\left\|\chi_{0}\right\|$. 
Now we start to investigate the asymptotic stability of the stationary solution. Here the stationary solution $(\tilde{\rho}, \tilde{u}, \tilde{E})(x)$ is a solution to the system (1.1) independent of a time variable $t$. Therefore, it satisfies the following stationary equations

$$
\left\{\begin{array}{l}
(\tilde{\rho} \tilde{u})_{x}=0 \\
\left(\tilde{\rho} \tilde{u}^{2}+p(\tilde{\rho})\right)_{x}=\mu \tilde{u}_{x x}+\tilde{\rho} \tilde{E}, \\
\tilde{E}_{x}=\tilde{\rho}-\rho_{+} .
\end{array}\right.
$$

The boundary conditions for $(\tilde{\rho}, \tilde{u}, \tilde{E})$ are assumed to be the same as the ones given in (1.2) and (1.3), namely

$$
\tilde{u}(0)=u_{b}<0,(\tilde{\rho}, \tilde{u}, \tilde{E})(\infty)=\left(\rho_{+}, u_{+}, 0\right), \inf _{x \in \mathbb{R}_{+}} \tilde{\rho}(x)>0 .
$$

One of our objectives is to give a sufficient condition which guarantees the existence of stationary solution to the outflow problem (1.1)-(1.3). To this end, we need to define a boundary strength $\delta$ as

$$
\delta:=\left|u_{b}-u_{+}\right|,
$$

which is used to measure the magnitude of the stationary wave $(\tilde{\rho}, \tilde{u}, \tilde{E})$. We introduce Mach number $M_{+}$at the spatial asymptotic state as

$$
M_{+}:=\frac{\left|u_{+}\right|}{c_{+}}
$$

where $c_{+}:=\sqrt{p^{\prime}\left(\rho_{+}\right)}$is sound speed. In particular, $M_{+}>1$ is referred to as the supersonic case at spatial infinity.

Theorem 1.1 (Existence of stationary waves). Suppose that $\delta<\varepsilon_{0}$ holds, where $\varepsilon_{0}$ is a certain positive constant. Then the stationary problem (1.5) and (1.6) has a unique smooth solution $(\tilde{\rho}, \tilde{u}, \tilde{E})$ which satisfies the following decay estimates

$$
\left|\partial_{x}^{k}\left(\tilde{\rho}(x)-\rho_{+}, \tilde{u}(x)-u_{+}, \tilde{E}(x)\right)\right| \leq C \delta e^{-c x}, \text { for } k=0,1,2, \ldots
$$

where $C$ and $c$ are positive constants.

Remark 1.1. If $\delta \neq 0$, then $\tilde{E}$ can not vanish. Furthermore, both $\tilde{\rho}$ and $\tilde{u}$ are nontrivial. This fact can be easily proved by using Lemma 1.1 in [10] and contradiction. In fact, the nontrivial profile $(\tilde{\rho}, \tilde{u}, \tilde{E})$ is called the boundary layer in some literature. Compared with the previous results, especially the ones in [5, 26], it should be pointed out that $\tilde{E} \neq 0$ is a fundamental difference.

Remark 1.2. Under the influence of electric field, there exists a boundary layer even for the case of $M_{+}<1$. Besides that, the boundary layer converges to the spatial asymptotic state at an exponential rate even for the case of $M_{+} \leq 1$. These are obviously different from the corresponding results in the absence of the electric field (cf. [10]). 
The time asymptotic stability of the stationary wave $(\tilde{\rho}, \tilde{u}, \tilde{E})$ constructed in Theorem 1.1 is summarized as follows.

Theorem 1.2 ( $L^{\infty}$-asymptotic stability). Let $\sigma \in(0,1)$. Suppose that $\left(\rho_{0}, u_{0}\right) \in \mathcal{B}^{1+\sigma}\left(\mathbb{R}_{+}\right) \times$ $\mathcal{B}^{2+\sigma}\left(\mathbb{R}_{+}\right)$holds. There exists a positive constant $\varepsilon_{1}$ such that if

$$
\left\|\left(\rho_{0}-\tilde{\rho}, u_{0}-\tilde{u}\right)\right\|_{1}+\left\|E_{0}-\tilde{E}\right\|+\delta<\varepsilon_{1},
$$

then the outflow problem (1.1)-(1.3) has a unique global solution $(\rho, u, E)$ satisfying

$$
\begin{gathered}
\rho \in \mathcal{B}^{1+\sigma / 2,1+\sigma}\left([0, T] \times \mathbb{R}_{+}\right), u \in \mathcal{B}^{1+\sigma / 2,2+\sigma}\left([0, T] \times \mathbb{R}_{+}\right), \\
(\rho-\tilde{\rho}, u-\tilde{u}) \in C\left([0, \infty) ; H^{1}\left(\mathbb{R}_{+}\right)\right), E-\tilde{E} \in C\left([0, \infty) ; L^{2}\left(\mathbb{R}_{+}\right)\right),
\end{gathered}
$$

for an arbitrary $T>0$. Furthermore, $(\rho, u, E)$ converges to the stationary solution $(\tilde{\rho}, \tilde{u}, \tilde{E})$ uniformly as time tends to infinity, namely

$$
\lim _{t \rightarrow \infty}\|(\rho, u, E)(t)-(\tilde{\rho}, \tilde{u}, \tilde{E})\|_{L^{\infty}}=0 .
$$

For the supersonic case, the convergence rate is shown as follows.

Theorem $1.3\left(L^{\infty}\right.$-convergence rate). Suppose that $M_{+}>1$ and the same conditions as in Theorem 1.2 hold.

(i) If the initial data satisfies $(1+x)^{\frac{\alpha}{2}}\left(\rho_{0}-\tilde{\rho}\right),(1+x)^{\frac{\alpha}{2}}\left(u_{0}-\tilde{u}\right),(1+x)^{\frac{\alpha}{2}}\left(E_{0}-\tilde{E}\right) \in L^{2}\left(\mathbb{R}_{+}\right)$ for a certain positive constant $\alpha$, then the solution $(\rho, u, E)$ to the outflow problem (1.1)-(1.3) satisfies the following decay estimate

$$
\|(\rho, u, E)(t)-(\tilde{\rho}, \tilde{u}, \tilde{E})\|_{L^{\infty}} \leq C(1+t)^{-\frac{\alpha}{2}} .
$$

(ii) If the initial data satisfies $e^{\frac{\zeta}{2} x}\left(\rho_{0}-\tilde{\rho}\right), e^{\frac{\zeta}{2} x}\left(u_{0}-\tilde{u}\right), e^{\frac{\zeta}{2} x}\left(E_{0}-\tilde{E}\right) \in L^{2}\left(\mathbb{R}_{+}\right)$for a certain positive constant $\zeta$, then there exists a positive constant $\alpha$ such that the solution $(\rho, u, E)$ to the outflow problem (1.1)-(1.3) satisfies the following decay estimate

$$
\|(\rho, u, E)(t)-(\tilde{\rho}, \tilde{u}, \tilde{E})\|_{L^{\infty}} \leq C e^{-\alpha t} .
$$

The remainder of the present paper is organized as follows. In Section 2, we discuss the existence of the stationary wave and present the proof of Theorem 1.1 with the aid of the stable manifold theory. Sections 3 is devoted to showing the asymptotic stability of the stationary wave. Specifically, we prove Theorem 1.2 by deriving $H^{1}$ a priori estimates of the perturbation variables combined with Hölder estimates. In Section 4, for the supersonic case, the convergence rate mentioned in Theorem 1.3 is obtained by a time and space weighted energy method.

\section{Existence of stationary solution}

In this section, we establish the existence of the stationary solution which approaches the spatial asymptotic state exponentially by the stable manifold theory. To this end, we reformulate the boundary value problem (1.5)-(1.6) into a $3 \times 3$ autonomous system of the first order ordinary differential equations. 


\subsection{Reformulation of stationary problem}

First of all, the dimensionless form of the stationary problem (1.5)-(1.6) is derived. By the equation (1.5a) and the boundary conditions (1.6), it is easy to see

$$
\tilde{\rho}(x) \tilde{u}(x)=\rho_{+} u_{+} .
$$

From (2.1), we have $u_{+}=\rho(0) u_{b} / \rho_{+}<0$. Hence, the coordinate transformation $\hat{x}:=x /\left|u_{+}\right|$ and the function transformation

$$
(\hat{\rho}, \hat{u}, \hat{E})(\hat{x}):=\left(\frac{1}{\rho_{+}} \tilde{\rho}, \frac{1}{\left|u_{+}\right|} \tilde{u}, \frac{1}{\left|u_{+}\right|} \tilde{E}\right)(x)
$$

are well defined. After a few straightforward calculations, the new equations for $(\hat{\rho}, \hat{u}, \hat{E})$ are

$$
\left\{\begin{array}{l}
(\hat{\rho} \hat{u})_{\hat{x}}=0, \\
\left(\hat{\rho} \hat{u}^{2}+\frac{1}{K \gamma M_{+}^{2}} p(\hat{\rho})\right)_{\hat{x}}=\frac{1}{\nu M_{+}^{2}} \hat{u}_{\hat{x} \hat{x}}+\hat{\rho} \hat{E}, \\
\hat{E}_{\hat{x}}=\rho_{+}(\hat{\rho}-1),
\end{array}\right.
$$

where for simplicity in notation $\nu=K \gamma \rho_{+}^{\gamma} / \mu$. The corresponding boundary conditions for the function $(\hat{\rho}, \hat{u}, \hat{E})$ are derived from $(1.6)$ as

$$
\hat{u}(0)=\frac{u_{b}}{\left|u_{+}\right|}<0,(\hat{\rho}, \hat{u}, \hat{E})(\infty)=(1,-1,0), \inf _{\hat{x} \in \mathbb{R}_{+}} \hat{\rho}(\hat{x})>0 .
$$

In the following, we attempt to perform some appropriate transformations of variables which bring the stationary problem (2.3)-(2.4) into the framework of a $3 \times 3$ autonomous system. This is precisely the basis for applying the manifold theory. Integrating the equation (2.3a) over $(x, \infty)$ and using $(2.4)$ leads to the following relation between $\hat{\rho}$ and $\hat{u}$

$$
\hat{\rho}(\hat{x})=-\frac{1}{\hat{u}(\hat{x})}
$$

Furthermore, the standard transformation of variable $\hat{\theta}:=\hat{u}_{\hat{x}}$ applied to (2.3) yields the equivalent autonomous system

$$
\left\{\begin{array}{l}
\hat{u}_{\hat{x}}=\hat{\theta} \\
\hat{\theta}_{\hat{x}}=\nu\left[\left(-\frac{1}{\hat{u}}\right)^{\gamma+1}-M_{+}^{2}\right] \hat{\theta}+\nu M_{+}^{2} \frac{\hat{E}}{\hat{u}}, \\
\hat{E}_{\hat{x}}=-\rho_{+}\left(\frac{1}{\hat{u}}+1\right) .
\end{array}\right.
$$

It is reasonable that the additional property $\hat{\theta}(\infty)=0$ is assumed in advance, since we hope that the stationary solution has some decay property. To discuss the solvability of (2.6) near the infinity asymptotic state $(-1,0,0)$, we need to introduce the stationary perturbation variables given by

$$
(\bar{u}, \bar{\theta}, \bar{E}):=(\hat{u}, \hat{\theta}, \hat{E})-(-1,0,0)
$$


Then (2.6) is transformed into the vector equations for $(\bar{u}, \bar{\theta}, \bar{E})$

$$
\frac{d}{d \hat{x}}\left(\begin{array}{c}
\bar{u} \\
\bar{\theta} \\
\bar{E}
\end{array}\right)=J\left(\begin{array}{c}
\bar{u} \\
\bar{\theta} \\
\bar{E}
\end{array}\right)+\left(\begin{array}{c}
0 \\
h_{2}(\bar{u}, \bar{\theta}, \bar{E}) \\
h_{3}(\bar{u})
\end{array}\right),
$$

where $J$ is the Jacobian matrix at an equilibrium point $(0,0,0)$ defined by

$$
J:=\left(\begin{array}{ccc}
0 & 1 & 0 \\
0 & \left(1-M_{+}^{2}\right) \nu & -\nu M_{+}^{2} \\
\rho_{+} & 0 & 0
\end{array}\right)
$$

and $h_{2}$ and $h_{3}$ are nonlinear terms that

$$
h_{2}(\bar{u}, \bar{\theta}, \bar{E}):=\nu\left[\left(-\frac{1}{\bar{u}-1}\right)^{\gamma+1}-1\right] \bar{\theta}+\nu M_{+}^{2} \bar{E}\left(1+\frac{1}{\bar{u}-1}\right), h_{3}(\bar{u}):=-\frac{\rho_{+} \bar{u}^{2}}{\bar{u}-1} .
$$

From the above procedure, the boundary conditions for $(\bar{u}, \bar{\theta}, \bar{E})$ are easy to be summed up as

$$
\bar{u}(0)=\bar{u}_{b}:=\frac{u_{b}}{\left|u_{+}\right|}+1,(\bar{u}, \bar{\theta}, \bar{E})(\infty)=(0,0,0) .
$$

\subsection{Proof of Theorem 1.1}

We are now in a position to prove Theorem 1.1.

Proof of Theorem 1.1. Let $\lambda_{1}, \lambda_{2}, \lambda_{3}$ designate all the eigenvalues which come from the characteristic equation

$$
\operatorname{det}(\lambda I-J)=\lambda^{3}-\nu\left(1-M_{+}^{2}\right) \lambda^{2}+\nu \rho_{+} M_{+}^{2}=0 .
$$

From Vieta's formulas, we have

$$
\begin{aligned}
& \lambda_{1} \lambda_{2} \lambda_{3}=-\nu \rho_{+} M_{+}^{2}<0, \\
& \lambda_{1} \lambda_{2}+\lambda_{1} \lambda_{3}+\lambda_{2} \lambda_{3}=0 .
\end{aligned}
$$

The inequality (2.13a) obviously implies the existence of a negative eigenvalue. Without the loss of generality, we assume $\lambda_{1}<0$. It remains to determine the symbols of $\operatorname{Re} \lambda_{2}$ and $\operatorname{Re} \lambda_{3}$. There are two cases to consider.

Case 1. $\lambda_{2}, \lambda_{3}$ are real roots. One easily shows from (2.13) that

$$
\lambda_{2} \lambda_{3}=-\frac{\nu \rho_{+} M_{+}^{2}}{\lambda_{1}}>0, \quad \lambda_{2}+\lambda_{3}=-\frac{\lambda_{2} \lambda_{3}}{\lambda_{1}}>0,
$$

which clearly imply that $\lambda_{2}>0$ and $\lambda_{3}>0$.

Case 2. $\lambda_{2}, \lambda_{3}$ are two complex conjugate roots. In this case, the expression (2.13b) is equivalent to the form

$$
\operatorname{Re} \lambda_{3}=\operatorname{Re} \lambda_{2}=-\frac{\left|\lambda_{2}\right|^{2}}{2 \lambda_{1}} .
$$

It is immediate from the symbol of $\lambda_{1}$ that the assertion $\operatorname{Re} \lambda_{3}=\operatorname{Re} \lambda_{2}>0$ holds. 
In order to make the manifold theory directly applicable, we need to reduce the equations (2.8) to block diagonal form. From elementary linear algebra, there is a real nonsingular matrix $P:=\left(p_{i j}\right)_{3 \times 3}$ such that

$$
P^{-1} J P=\operatorname{diag}\left(\lambda_{1}, A\right)
$$

where $A$ is a $2 \times 2$ matrix having eigenvalues with positive real part. Therefore, the linear transformation

$$
\left(\begin{array}{c}
\mathrm{U} \\
\Theta \\
\Sigma
\end{array}\right):=P^{-1}\left(\begin{array}{c}
\bar{u} \\
\bar{\theta} \\
\bar{E}
\end{array}\right)
$$

applied to (2.8) and (2.11) yields the equivalent boundary value problem

$$
\left\{\begin{array}{l}
\frac{d}{d \hat{x}}\left(\begin{array}{c}
\mathrm{U} \\
\Theta \\
\Sigma
\end{array}\right)=\operatorname{diag}\left(\lambda_{1}, A\right)\left(\begin{array}{c}
\mathrm{U} \\
\Theta \\
\Sigma
\end{array}\right)+\left(\begin{array}{c}
\mathrm{H}_{1}(\mathrm{U}, \Theta, \Sigma) \\
\mathrm{H}_{2}(\mathrm{U}, \Theta, \Sigma) \\
\mathrm{H}_{3}(\mathrm{U}, \Theta, \Sigma)
\end{array}\right) \\
p_{11} \mathrm{U}(0)+p_{12} \Theta(0)+p_{13} \Sigma(0)=\bar{u}_{b}, \quad\left(\begin{array}{lll}
\mathrm{U} & \Theta & \Sigma
\end{array}\right)(\infty)=\left(\begin{array}{lll}
0 & 0 & 0
\end{array}\right)
\end{array}\right.
$$

where $\mathrm{H}_{1}, \mathrm{H}_{2}$ and $\mathrm{H}_{3}$ are defined by

$$
\left(\begin{array}{c}
\mathrm{H}_{1}(\mathrm{U}, \Theta, \Sigma) \\
\mathrm{H}_{2}(\mathrm{U}, \Theta, \Sigma) \\
\mathrm{H}_{3}(\mathrm{U}, \Theta, \Sigma)
\end{array}\right):=P^{-1}\left(\begin{array}{c}
0 \\
h_{2}(\bar{u}, \bar{\theta}, \bar{E}) \\
h_{3}(\bar{u})
\end{array}\right) .
$$

For the sake of technique only, it is convenient to introduce two undetermined parameters $\bar{\theta}_{b}:=\bar{\theta}(0)$ and $\bar{E}_{b}:=\bar{E}(0)$, simultaneously add the auxiliary boundary conditions $p_{21} \mathrm{U}(0)+$ $p_{22} \Theta(0)+p_{23} \Sigma(0)=\bar{\theta}_{b}$ and $p_{31} \mathrm{U}(0)+p_{32} \Theta(0)+p_{33} \Sigma(0)=\bar{E}_{b}$ which are combined with the first part of (2.18b) and described very succinctly as

$$
\left(\begin{array}{c}
\mathrm{U}(0) \\
\Theta(0) \\
\Sigma(0)
\end{array}\right)=P^{-1}\left(\begin{array}{c}
\bar{u}_{b} \\
\bar{\theta}_{b} \\
\bar{E}_{b}
\end{array}\right)
$$

Since the previous argument proceeds inductively to yield the fact that $J$ has one negative eigenvalue $\lambda_{1}$ as well as two eigenvalues with positive real part. By virtue of the manifold theory in [24], there exist a $C^{\infty}$ local stable manifold $W_{l o c}^{s}(0,0,0)$ and a $C^{\infty}$ local unstable manifold $W_{l o c}^{u}(0,0,0)$ corresponding to $\lambda_{1}$ and the other eigenvalues, respectively. More specifically, $W_{l o c}^{s}(0,0,0)$ can locally be represented by a graph over the $\mathrm{U}$ variables, i.e.,

$$
\begin{aligned}
W_{l o c}^{s}(0,0,0)= & \left\{(\mathrm{U}, \Theta, \Sigma) \in \mathbb{R}^{3} \mid \exists C^{\infty} \text { functions } h_{\Theta}^{s} \text { and } h_{\Sigma}^{s} \text { s.t. } \Theta=h_{\Theta}^{s}(\mathrm{U})\right. \\
& \Sigma=h_{\Sigma}^{s}(\mathrm{U}) \text { with } h_{\Theta}^{s}(0)=D h_{\Theta}^{s}(0)=0, h_{\Sigma}^{s}(0)=D h_{\Sigma}^{s}(0)=0 \\
& \text { for }|\mathrm{U}| \text { sufficiently small }\} .
\end{aligned}
$$


Furthermore, if $(\mathrm{U}(0), \Theta(0), \Sigma(0))$ is located on the stable manifold $W_{l o c}^{s}(0,0,0)$, then the problem (2.18) and (2.20) has a unique smooth solution $(\mathrm{U}, \Theta, \Sigma)$ which approaches the origin $(0,0,0)$ at an exponential rate asymptotically as $\hat{x} \rightarrow \infty$, i.e.,

$$
\left|\partial_{\hat{x}}^{k}(\mathrm{U}(\hat{x}), \Theta(\hat{x}), \Sigma(\hat{x}))\right| \leq C|\mathrm{U}(0)| e^{-c \hat{x}}, \text { for } k=0,1,2, \ldots
$$

Next we assert that if $(\mathrm{U}(0), \Theta(0), \Sigma(0)) \in\left\{(\mathrm{U}, \Theta, \Sigma) \in \mathbb{R}^{3} \mid \Theta=h_{\Theta}^{s}(\mathrm{U}), \quad \Sigma=h_{\Sigma}^{s}(\mathrm{U})\right\}$, the original stationary problem (1.5) and (1.6) with $\delta \ll 1$ is equivalent to the boundary value problem (2.18) and (2.20) with $|U(0)| \ll 1$. It suffices to show that $\mathrm{U}(0)$ depends locally and only on the original data $u_{b}$ in a continuous differentiable way. In fact, by premultiplying both sides of the equality $(2.16)$ by $P$ so as to cancel the matrix $P^{-1}$, we immediately deduce that $J P=P \operatorname{diag}\left(\lambda_{1}, A\right)$ including the algebraic equations for $\left(p_{11}, p_{21}, p_{31}\right)$

$$
\left\{\begin{array}{l}
p_{21}=\lambda_{1} p_{11}, \\
\rho_{+} p_{11}=\lambda_{1} p_{31}
\end{array}\right.
$$

according to the definition of matrix multiplication. From (2.23), $p_{21}$ and $p_{31}$ must be equal to zero as long as $p_{11}=0$. On the other hand, this is impossible since the matrix $P$ is nonsingular. This contradiction shows that the fact $p_{11} \neq 0$ holds. Here the structure of the matrix $J$ plays an important role. Notice that $(\mathrm{U}(0), \Theta(0), \Sigma(0)) \in\left\{(\mathrm{U}, \Theta, \Sigma) \in \mathbb{R}^{3} \mid \Theta=h_{\Theta}^{s}(\mathrm{U}), \Sigma=h_{\Sigma}^{s}(\mathrm{U})\right\}$, therefore the first part of (2.18) can be rewritten as

$$
p_{11} \mathrm{U}(0)+p_{12} h_{\Theta}^{s}(\mathrm{U}(0))+p_{13} h_{\Sigma}^{s}(\mathrm{U}(0))=\bar{u}_{b}
$$

Since $p_{11} \neq 0$, by using the implicit function theorem, one easily solves the equation (2.24) for $\mathrm{U}(0)$ to obtain a unique $C^{1}$ function of $\bar{u}_{b}$ in a neighborhood of the origin $\bar{u}_{b}=0$. Thus, not only is the useful inequality

$$
c \delta<|\mathrm{U}(0)|<C \delta, \quad \text { if }|\delta| \ll 1
$$

true by using the differential mean value theorem and (2.11), but also the parameters $\bar{\theta}_{b}$ and $\bar{E}_{b}$ are uniquely determined by $u_{b}$ from (2.11), (2.20) and (2.21). This implies the assertion mentioned at the beginning of this paragraph holds. In addition, from (2.25), it follows that the condition (2.22) is also equivalent to (1.9).

By combining the information as above, we complete the proof of Theorem 1.1.

Remark 2.1. It is worth to point out that with almost the same proof, we can also obtain a similar existence result for the corresponding inflow problem.

\section{Asymptotic stability of the stationary solution}

This section is devoted to proving the asymptotic stability of the stationary solution to the outflow problem (1.1)-(1.3), which is stated in Theorem 1.2. 


\subsection{Local existence and Poincaré type inequality}

We regard the solution $(\rho, u, E)$ as a perturbation from the stationary solution $(\tilde{\rho}, \tilde{u}, \tilde{E})$ and introduce the new unknown functions as

$$
(\varphi, \psi, \chi)(t, x):=(\rho, u, E)(t, x)-(\tilde{\rho}, \tilde{u}, \tilde{E})(x) .
$$

In what follows we derive the equation in terms of the perturbation variables $(\varphi, \psi, \chi)$. Subtracting (1.5) from (1.1) yields that

$$
\left\{\begin{array}{l}
\varphi_{t}+u \varphi_{x}+\rho \psi_{x}=f \\
\rho\left(\psi_{t}+u \psi_{x}\right)+p^{\prime}(\rho) \varphi_{x}-\mu \psi_{x x}=g+\rho \chi+\tilde{E} \varphi \\
\chi_{x}=\varphi
\end{array}\right.
$$

where

$$
\begin{gathered}
f:=-\left(\tilde{\rho}_{x} \psi+\tilde{u}_{x} \varphi\right) \\
g:=-(\varphi \psi+\tilde{u} \varphi+\tilde{\rho} \psi) \tilde{u}_{x}-\left(p^{\prime}(\rho)-p^{\prime}(\tilde{\rho})\right) \tilde{\rho}_{x} .
\end{gathered}
$$

The corresponding initial and boundary conditions are derived from (1.2), (1.3) and (1.6) as follows

$$
\begin{gathered}
(\varphi, \psi)(0, x)=\left(\varphi_{0}, \psi_{0}\right)(x):=\left(\rho_{0}-\tilde{\rho}, u_{0}-\tilde{u}\right)(x), \\
\psi(t, 0)=0, \chi(t, \infty)=0 .
\end{gathered}
$$

For convenience, we define the solution space $X(0, T)$ by

$$
\begin{gathered}
X(0, T):=\left\{(\varphi, \psi, \chi) \mid \varphi \in \mathcal{B}^{1+\sigma / 2,1+\sigma}\left([0, T] \times \mathbb{R}_{+}\right), \psi \in \mathcal{B}^{1+\sigma / 2,2+\sigma}\left([0, T] \times \mathbb{R}_{+}\right),\right. \\
(\varphi, \psi) \in C\left([0, T] ; H^{1}\left(\mathbb{R}_{+}\right)\right), \chi \in C\left([0, T] ; L^{2}\left(\mathbb{R}_{+}\right)\right), \\
\left.\varphi_{x} \in L^{2}\left(0, T ; L^{2}\left(\mathbb{R}_{+}\right)\right), \psi_{x} \in L^{2}\left(0, T ; H^{1}\left(\mathbb{R}_{+}\right)\right)\right\},
\end{gathered}
$$

for $T>0,0<\sigma<1$.

Next we state a local existence result, which is proved by a standard iteration method (cf. [20]). The details of the proof are omitted here.

Lemma 3.1. Suppose the same conditions as in Theorem 1.2 hold. Then there exists a positive constant $T_{0}$, depending only on $\left|\varphi_{0}\right|_{\mathcal{B}^{1+\sigma}}$ and $\left|\psi_{0}\right|_{\mathcal{B}^{2+\sigma}}$, such that the perturbation problem (3.2) and (3.4) has a unique solution $(\varphi, \psi, \chi) \in X\left(0, T_{0}\right)$.

We derive the uniform a priori estimate by the following technical lemma, which is called the Poincaré type inequality.

Lemma 3.2. For any function $v$ and $k=1,2$, there exists a positive constant $C$ such that

$$
\begin{aligned}
& \int_{0}^{t} \int_{0}^{\infty}\left(\left|\partial_{x}^{k} \tilde{u}\right||v|^{2}\right)(\tau, x) d x d \tau \leq C \delta \int_{0}^{t}\left(|v(\tau, 0)|^{2}+\left\|v_{x}(\tau)\right\|^{2}\right) d \tau \\
& \int_{0}^{t} \int_{0}^{\infty}\left(|\tilde{E}||v|^{2}\right)(\tau, x) d x d \tau \leq C \delta \int_{0}^{t}\left(|v(\tau, 0)|^{2}+\left\|v_{x}(\tau)\right\|^{2}\right) d \tau
\end{aligned}
$$

provided that the right hand side of (3.5) is bounded. 
Proof. We only prove (3.5b) for the presence of the electric field $\tilde{E}$. In fact, it is clear that

$$
v(\tau, x)=v(\tau, 0)+\int_{0}^{x} v_{x}(\tau, y) d y .
$$

Notice that the inequality $\left|\int_{0}^{x} v_{x}(\tau, y) d y\right| \leq \sqrt{x}\left\|v_{x}(\tau)\right\|$ holds. From the identity (3.6), it follows that

$$
\int_{0}^{\infty}\left(|\tilde{E}||v|^{2}\right)(\tau, x) d x \leq C \int_{0}^{\infty}|\tilde{E}|\left(|v(\tau, 0)|^{2}+x|| v_{x}(\tau)||^{2}\right) d x
$$

Substituting (1.9) in (3.7) and integrating the resultant inequality from 0 to $t$ yields the desired estimate (3.5b). Here we have used the fact $\int_{0}^{\infty} x e^{-c x} d x<\infty$.

The proof of $(3.5 \mathrm{a})$ is similar and thus is omitted for brevity.

\section{$3.2 \quad$ A priori estimates}

To extend the local solution to a global solution, we need to establish the uniform a priori estimate of the perturbation variables $(\varphi, \psi, \chi)$ in the space $H^{1}$. The result is given in the following proposition.

For simplicity, we use notations

$$
\begin{gathered}
N(t):=\sup _{0 \leq \tau \leq t}\left(\|(\varphi, \psi)(\tau)\|_{1}+\|\chi(\tau)\|\right), \\
M^{2}(t):=\|\varphi(t)\|_{1}^{2}+\left\|\psi_{x}(t)\right\|_{1}^{2}+\left|\left(\varphi, \varphi_{x}\right)(t, 0)\right|^{2}+|\chi(t, 0)|^{2} .
\end{gathered}
$$

Proposition 3.3. Suppose that the same conditions as in Theorem 1.2 hold. Let $(\varphi, \psi, \chi) \in$ $X(0, T)$ be a solution to the perturbation problem (3.2) and (3.4) for some positive $T$. There exist positive constants $\varepsilon_{2}$ and $C$ independent of $T$ such that if $N(T)+\delta<\varepsilon_{2}$, then the solution $(\varphi, \psi, \chi)$ satisfies the estimate

$$
\|(\varphi, \psi)(t)\|_{1}+\|\chi(t)\|+\int_{0}^{t} M^{2}(\tau) d \tau \leq C\left(\left\|\left(\varphi_{0}, \psi_{0}\right)\right\|_{1}+\left\|\chi_{0}\right\|\right),
$$

for an arbitrary $t \in[0, T]$.

Proof. The proof is divided into three steps, which are stated in Lemmas 3.4, 3.5 and 3.6, respectively. Then combining the uniform estimates proved in the above lemmas yields the desired estimate (3.8).

Notice that (1.9) implies

$$
|\tilde{u}| \leq C, \tilde{\rho} \leq C
$$

From the equation (2.1), it follows that

$$
0<c \leq \tilde{\rho},-\tilde{u} \leq C
$$

By the Sobolev inequality, we have

$$
\|\varphi(t)\|_{L^{\infty}} \leq C\|\varphi(t)\|^{\frac{1}{2}}\left\|\varphi_{x}(t)\right\|^{\frac{1}{2}} \leq C N(t),
$$


for any $0 \leq t \leq T$. The smallness assumption on $N(T)$ in Proposition 3.3 ensures that if $\varepsilon_{2}$ is sufficiently small, then there exist certain positive constants $c$ and $C$ such that for any $t \in[0, T], x \in \mathbb{R}_{+}$,

$$
0<c \leq \rho(t, x) \leq C
$$

Similarly,

$$
|u(t, x)| \leq C,|E(t, x)| \leq C,
$$

for $t \in[0, T], x \in \mathbb{R}_{+}$. From (3.11), $f$ and $g$ in (3.3) are estimated as

$$
|f|,|g| \leq C\left|\tilde{u}_{x}\right||(\varphi, \psi)|
$$

The basic energy estimate is shown as follows.

Lemma 3.4. There exist positive constants $\varepsilon_{3}$ and $C$ such that if $N(T)+\delta<\varepsilon_{3}$, then it holds that

$$
\begin{aligned}
\|(\varphi, \psi, \chi)(t)\|^{2} & +\int_{0}^{t}\left(\left\|\psi_{x}(\tau)\right\|^{2}+|\varphi(\tau, 0)|^{2}+|\chi(\tau, 0)|^{2}\right) d \tau \\
& \leq C\left\{\left\|\left(\varphi_{0}, \psi_{0}, \chi_{0}\right)\right\|^{2}+(N(t)+\delta) \int_{0}^{t} M^{2}(\tau) d \tau\right\},
\end{aligned}
$$

for an arbitrary $t \in[0, T]$.

Proof. Multiplying (3.2b) by $\psi$, then substituting $\psi_{x}=\left(f-\varphi_{t}-u \varphi_{x}\right) / \rho$ and using (3.2c), (1.4), (3.9) and (3.11), one has

$$
\begin{aligned}
& \frac{1}{2}\left(\rho \psi^{2}+\chi^{2}+\frac{p^{\prime}(\rho)}{\rho} \varphi^{2}\right)_{t}+\left(\rho u \frac{\psi^{2}}{2}+p^{\prime}(\rho) \varphi \psi+\frac{p^{\prime}(\rho)}{\rho} u \frac{\varphi^{2}}{2}+\frac{\tilde{u}}{2} \chi^{2}+\mu \psi \psi_{x}\right)_{x}+\mu\left(\psi_{x}\right)^{2} \\
& =R_{1}+R_{2}
\end{aligned}
$$

where

$$
\begin{aligned}
R_{1}:= & \frac{1}{2} \tilde{u}_{x} \chi^{2}+\tilde{E} \varphi \psi-p^{\prime \prime}(\rho) \tilde{u}_{x} \frac{\varphi^{2}}{2}-\frac{p^{\prime}(\rho)}{\rho} \tilde{\rho}_{x} \varphi \psi+p^{\prime \prime}(\rho) \tilde{\rho}_{x} \varphi \psi-\tilde{u} \tilde{u}_{x} \varphi \psi \\
& -\rho \tilde{u}_{x} \psi^{2}-\left(p^{\prime}(\rho)-p^{\prime}(\tilde{\rho})\right) \tilde{\rho}_{x} \psi \\
R_{2}:= & p^{\prime \prime}(\rho) \varphi \varphi_{x} \psi+\frac{p^{\prime}(\rho)}{\rho} \psi_{x} \frac{\varphi^{2}}{2}-p^{\prime \prime}(\rho) \psi_{x} \frac{\varphi^{2}}{2} .
\end{aligned}
$$

The Schwarz inequality, the differential mean value theorem, (1.4), (1.5a), (3.10) and (3.11) guarantee

$$
\left|R_{1}\right| \leq C|(\varphi, \psi, \chi)|^{2}\left(\left|\tilde{u}_{x}\right|+|\tilde{E}|\right) .
$$

Integrating (3.15) over the region $[0, t] \times \mathbb{R}_{+}$and using the boundary condition (3.4b), we have

$$
\begin{aligned}
& \frac{1}{2} \int_{0}^{\infty}\left(\rho \psi^{2}+\chi^{2}+\frac{p^{\prime}(\rho)}{\rho} \varphi^{2}\right)(t, x) d x-\frac{u_{b}}{2} \int_{0}^{t}\left(\frac{p^{\prime}(\rho)}{\rho} \varphi^{2}+\chi^{2}\right)(\tau, 0) d \tau+\mu \int_{0}^{t}\left\|\psi_{x}(\tau)\right\|^{2} d \tau \\
& =\frac{1}{2} \int_{0}^{\infty}\left(\rho \psi^{2}+\chi^{2}+\frac{p^{\prime}(\rho)}{\rho} \varphi^{2}\right)(0, x) d x+\int_{0}^{t} \int_{0}^{\infty} R_{1}(\tau, x) d x d \tau+\int_{0}^{t} \int_{0}^{\infty} R_{2}(\tau, x) d x d \tau
\end{aligned}
$$


The second term on the left hand side of (3.18) is handled by using $u_{b}<0,(1.4)$ and (3.11) as

$$
-\frac{u_{b}}{2} \int_{0}^{t}\left(\frac{p^{\prime}(\rho)}{\rho} \varphi^{2}+\chi^{2}\right)(\tau, 0) d \tau \geq c \int_{0}^{t}\left(|\varphi(\tau, 0)|^{2}+|\chi(\tau, 0)|^{2}\right) d \tau .
$$

Due to (3.17) and the Poincaré type inequality (3.5), the second term on the right hand side of (3.18) is estimated as

$$
\begin{gathered}
\left|\int_{0}^{t} \int_{0}^{\infty} R_{1}(\tau, x) d x d \tau\right| \leq C \delta \int_{0}^{t}\left(|| \psi_{x}(\tau)\left\|^{2}+|| \varphi_{x}(\tau)\right\|^{2}+\|\varphi(\tau)\|^{2}\right. \\
\left.+|\varphi(\tau, 0)|^{2}+|\chi(\tau, 0)|^{2}\right) d \tau
\end{gathered}
$$

By using the Sobolev inequality, the Schwarz inequality, (3.16b), (1.4) and (3.11), we have

$$
\left|\int_{0}^{t} \int_{0}^{\infty} R_{2}(\tau, x) d x d \tau\right| \leq C N(t) \int_{0}^{t}\left(\left\|\psi_{x}(\tau)\right\|^{2}+\left\|\varphi_{x}(\tau)\right\|^{2}+\|\varphi(\tau)\|^{2}\right) d \tau .
$$

Substitution of the estimates (3.19)-(3.21) in (3.18) completes the proof.

Next, we derive the $L^{2}$ estimate for the first-order derivatives of the perturbation variables. As the existence of the higher-order derivatives of the solution is not supposed, we need to use the difference quotient for the rigorous derivation of the higher-order estimates. By the techniques used by S. Kawashima, S. Nishibata and P. Zhu in [10], we may verify

$$
\begin{gathered}
(\varphi, \psi) \in C\left([0, T], H^{2}\left(\mathbb{R}_{+}\right)\right), \varphi_{x} \in L^{2}\left(0, T ; H^{1}\left(\mathbb{R}_{+}\right)\right) \\
\psi_{x} \in L^{2}\left(0, T ; H^{2}\left(\mathbb{R}_{+}\right)\right), \chi_{x} \in L^{2}\left(0, T ; L^{2}\left(\mathbb{R}_{+}\right)\right)
\end{gathered}
$$

Lemma 3.5. There exist positive constants $\varepsilon_{4}\left(\leq \varepsilon_{3}\right)$ and $C$ such that if $N(T)+\delta<\varepsilon_{4}$, then it holds that

$$
\begin{aligned}
\left\|\varphi_{x}(t)\right\|^{2} & +\int_{0}^{t}\left(\|\varphi(\tau)\|_{1}^{2}+\left|\varphi_{x}(\tau, 0)\right|^{2}\right) d \tau \\
& \leq C\left\{\left\|\left(\varphi_{0}, \psi_{0}\right)\right\|_{1}^{2}+\left\|\chi_{0}\right\|^{2}+(N(T)+\delta) \int_{0}^{t} M^{2}(\tau) d \tau\right\},
\end{aligned}
$$

for an arbitrary $t \in[0, T]$.

Proof. The proof of (3.22) will consist of the following two steps. Firstly, differentiating the equation (3.2a) in $x$, dividing it by $\rho$, and using (1.1a), one has

$$
\left(\frac{\varphi_{x}}{\rho}\right)_{t}+u\left(\frac{\varphi_{x}}{\rho}\right)_{x}+\psi_{x x}=Q
$$

where

$$
Q:=\frac{f_{x}-\rho_{x} \psi_{x}}{\rho}=-\frac{\left(\tilde{u}_{x} \varphi+\tilde{\rho}_{x} \psi\right)_{x}+\rho_{x} \psi_{x}}{\rho} .
$$

Then multiplying $(3.23)$ by $\varphi_{x} / \rho$ yields

$$
\frac{1}{2}\left[\left(\frac{\varphi_{x}}{\rho}\right)^{2}\right]_{t}+\frac{u}{2}\left[\left(\frac{\varphi_{x}}{\rho}\right)^{2}\right]_{x}+\frac{\varphi_{x} \psi_{x x}}{\rho}=\frac{\varphi_{x} Q}{\rho} .
$$


Secondly, we expect to eliminate the term $\left(\varphi_{x} \psi_{x x}\right) / \rho$ in $(3.25)$. Multiply $(3.2 \mathrm{~b})$ by $\varphi_{x} / \rho$ to get that

$$
\left(\psi \varphi_{x}\right)_{t}-\left(\psi \varphi_{t}\right)_{x}+\left(\varphi_{t}+u \varphi_{x}\right) \psi_{x}+\frac{p^{\prime}(\rho)}{\rho} \varphi_{x}^{2}-\mu \frac{\psi_{x x} \varphi_{x}}{\rho}=\frac{g \varphi_{x}}{\rho}+\chi \varphi_{x}+\frac{\tilde{E} \varphi \varphi_{x}}{\rho} .
$$

Here we have used the identity

$$
\psi_{t} \varphi_{x}=\left(\psi \varphi_{x}\right)_{t}-\psi \varphi_{x t}=\left(\psi \varphi_{x}\right)_{t}-\left(\psi \varphi_{t}\right)_{x}+\psi_{x} \varphi_{t}
$$

Multiplying (3.25) by $\mu$ and adding the resultant equation to (3.26), we have

$$
\begin{aligned}
& {\left[\frac{\mu}{2}\left(\frac{\varphi_{x}}{\rho}\right)^{2}+\psi \varphi_{x}\right]_{t}+\left[\frac{\mu}{2} u\left(\frac{\varphi_{x}}{\rho}\right)^{2}-\psi \varphi_{t}\right]_{x}-\frac{\mu}{2} u_{x}\left(\frac{\varphi_{x}}{\rho}\right)^{2}+\frac{p^{\prime}(\rho)}{\rho} \varphi_{x}^{2}} \\
& =\mu Q \frac{\varphi_{x}}{\rho}-\left(\varphi_{t}+u \varphi_{x}\right) \psi_{x}+\frac{g \varphi_{x}}{\rho}+\chi \varphi_{x}+\frac{\tilde{E} \varphi_{x}}{\rho} .
\end{aligned}
$$

Substituting $\varphi_{t}+u \varphi_{x}=f-\rho \psi_{x}$ in (3.28), integrating the resultant equation over $[0, t] \times \mathbb{R}_{+}$ and using the boundary conditions $(1.3 \mathrm{a})$ and $(3.4 \mathrm{~b})$ yields

$$
\begin{aligned}
& \frac{\mu}{2} \int_{0}^{\infty}\left(\frac{\varphi_{x}}{\rho}\right)^{2}(t, x) d x+\int_{0}^{\infty}\left(\psi \varphi_{x}\right)(t, x) d x+\frac{\mu\left|u_{b}\right|}{2} \int_{0}^{t}\left(\frac{\varphi_{x}}{\rho}\right)^{2}(\tau, 0) d \tau \\
& -\frac{\mu}{2} \int_{0}^{t} \int_{0}^{\infty}\left(u_{x}\left(\frac{\varphi_{x}}{\rho}\right)^{2}\right)(\tau, x) d x d \tau+\int_{0}^{t} \int_{0}^{\infty}\left(\frac{p^{\prime}(\rho)}{\rho} \varphi_{x}^{2}\right)(\tau, x) d x d \tau \\
& =\frac{\mu}{2} \int_{0}^{\infty}\left(\frac{\varphi_{x}}{\rho}\right)^{2}(0, x) d x+\int_{0}^{\infty}\left(\psi \varphi_{x}\right)(0, x) d x+\int_{0}^{t} \int_{0}^{\infty}\left(\frac{\mu Q+g}{\rho} \varphi_{x}-f \psi_{x}\right)(\tau, x) d x d \tau \\
& \quad+\int_{0}^{t} \int_{0}^{\infty}\left(\rho \psi_{x}^{2}\right)(\tau, x) d x d \tau+\int_{0}^{t} \int_{0}^{\infty}\left(\chi \varphi_{x}\right)(\tau, x) d x d \tau+\int_{0}^{t} \int_{0}^{\infty} \frac{\tilde{E} \varphi \varphi_{x}}{\rho}(\tau, x) d x d \tau .
\end{aligned}
$$

We estimate the terms in (3.29) one by one. The second term on the left hand side is estimated by the Schwarz inequality as

$$
\left|\int_{0}^{\infty}\left(\psi \varphi_{x}\right)(t, x) d x\right| \leq C_{\epsilon}\|\psi(t)\|^{2}+\epsilon\left\|\varphi_{x}(t)\right\|^{2}
$$

where $\epsilon$ is an arbitrary positive constant and $C_{\epsilon}$ is a constant depending only on $\epsilon$. Note that $\|\psi\|^{2}$ has been estimated in (3.14). Substitute $u_{x}=\tilde{u}_{x}+\psi_{x}$, and then use (1.9), the Sobolev inequality $\left|\psi_{x}(\tau)\right| \leq C\left\|\psi_{x}(\tau)\right\|_{1}$ as well as the Schwarz inequality to get

$$
\begin{aligned}
\left|\int_{0}^{t} \int_{0}^{\infty}\left(u_{x}\left(\frac{\varphi_{x}}{\rho}\right)^{2}\right)(\tau, x) d x d \tau\right| & \leq \int_{0}^{t} \int_{0}^{\infty}\left(\left|\tilde{u}_{x}\right|\left|\frac{\varphi_{x}}{\rho}\right|^{2}+\left|\psi_{x}\right|\left|\frac{\varphi_{x}}{\rho}\right|^{2}\right)(\tau, x) d x d \tau \\
& \leq C \delta \int_{0}^{t}\left\|\varphi_{x}(\tau)\right\|^{2} d \tau+C \int_{0}^{t}\left\|\psi_{x}(\tau)\right\|_{1}\left\|\varphi_{x}(\tau)\right\|^{2} d \tau \\
& \leq C \delta \int_{0}^{t}\left\|\varphi_{x}(\tau)\right\|^{2} d \tau+C N(t) \int_{0}^{t}\left\|\psi_{x}(\tau)\right\|\left\|_{1}\right\| \varphi_{x}(\tau) \| d \tau \\
& \leq C \delta \int_{0}^{t}\left\|\varphi_{x}(\tau)\right\|^{2} d \tau+C N(t) \int_{0}^{t}\left(\left\|\psi_{x}(\tau)\right\|_{1}^{2}+\left\|\varphi_{x}(\tau)\right\|^{2}\right) d \tau \\
& \leq C(\delta+N(t)) \int_{0}^{t}\left(\left\|\psi_{x}(\tau)\right\|_{1}^{2}+\left\|\varphi_{x}(\tau)\right\|^{2}\right) d \tau .
\end{aligned}
$$


From (1.4) and (3.11), it follows that

$$
\int_{0}^{t} \int_{0}^{\infty}\left(\frac{p^{\prime}(\rho)}{\rho} \varphi_{x}^{2}\right)(\tau, x) d x d \tau \geq c \int_{0}^{t}\left\|\varphi_{x}(\tau)\right\|^{2} d \tau
$$

The Schwarz inequality guarantee

$$
\left|\int_{0}^{\infty}\left(\psi \varphi_{x}\right)(0, x) d x\right| \leq C\left(\left\|\psi_{0}\right\|^{2}+\left\|\varphi_{0 x}\right\|^{2}\right)
$$

From (1.5a) and (3.24), we obtain

$$
\begin{aligned}
|Q| & \leq C\left(\left|\tilde{u}_{x}\right|\left|\varphi_{x}\right|+\left|\tilde{u}_{x x}\right||\varphi|+\left|\tilde{\rho}_{x}\right|\left|\psi_{x}\right|+\left|\tilde{\rho}_{x x}\right||\psi|+\left|\varphi_{x}\right|\left|\psi_{x}\right|\right) \\
& \leq C\left(|(\varphi, \psi)|\left|\tilde{u}_{x x}\right|+|\psi|\left|\tilde{u}_{x}\right|+\left|\left(\varphi_{x}, \psi_{x}\right)\right|\left|\tilde{u}_{x}\right|+\left|\varphi_{x}\right|\left|\psi_{x}\right|\right) .
\end{aligned}
$$

Thus, by using the above estimate, (3.11), (3.13), (1.9), (3.5a) and the Sobolev inequality, the third term on the right hand side of (3.29) is estimated as

$$
\begin{aligned}
& \left|\int_{0}^{t} \int_{0}^{\infty}\left(\frac{\mu Q+g}{\rho} \varphi_{x}-f \psi_{x}\right)(\tau, x) d x d \tau\right| \\
& \leq C \int_{0}^{t} \int_{0}^{\infty}\left\{\left(|(\varphi, \psi)|\left|\tilde{u}_{x x}\right|+\left|\left(\varphi_{x}, \psi_{x}\right)\right|\left|\tilde{u}_{x}\right|+\left|\varphi_{x}\right|\left|\psi_{x}\right|+|(\varphi, \psi)|\left|\tilde{u}_{x}\right|\right)\left|\varphi_{x}\right|\right. \\
& \left.\quad+|(\varphi, \psi)|\left|\tilde{u}_{x}\right|\left|\psi_{x}\right|\right\}(\tau, x) d x d \tau \\
& \leq C(\delta+N(t)) \int_{0}^{t}\left(|| \psi_{x}(\tau)\left\|_{1}^{2}+\right\| \varphi_{x}(\tau) \|^{2}+|\varphi(\tau, 0)|^{2}\right) d \tau .
\end{aligned}
$$

Due to (3.11), we have

$$
\left|\int_{0}^{t} \int_{0}^{\infty}\left(\rho \psi_{x}^{2}\right)(\tau, x) d x d \tau\right| \leq C \int_{0}^{t}\left\|\psi_{x}(\tau)\right\|^{2} d \tau
$$

Note that $\int_{0}^{t}\left\|\psi_{x}(\tau)\right\|^{2} d \tau$ has been estimated in (3.14). By applying integration by parts, (3.2c) and $(3.4 \mathrm{~b})$, the fifth term on the right hand side of (3.29) is rewritten as

$$
\int_{0}^{t} \int_{0}^{\infty}\left(\chi \varphi_{x}\right)(\tau, x) d x d \tau=-\int_{0}^{t}(\chi \varphi)(\tau, 0) d \tau-\int_{0}^{t}\|\varphi(\tau)\|^{2} d \tau
$$

Furthermore, we have

$$
\left|\int_{0}^{t}(\chi \varphi)(\tau, 0) d \tau\right| \leq C\left(\int_{0}^{t}|\chi(\tau, 0)|^{2} d \tau+\int_{0}^{t}|\varphi(\tau, 0)|^{2} d \tau\right) .
$$

Note that the above two terms have been estimated in (3.14). The last term in (3.29) is estimated by using (3.11), (1.9) and the Schwarz inequality as

$$
\begin{aligned}
\left|\int_{0}^{t} \int_{0}^{\infty}\left(\frac{\varphi}{\rho} \varphi_{x} \tilde{E}\right)(\tau, x) d x d \tau\right| & \leq C \int_{0}^{t} \int_{0}^{\infty}\left(|\tilde{E}|\left(|\varphi|^{2}+\left|\varphi_{x}\right|^{2}\right)\right)(\tau, x) d x d \tau \\
& \leq C \delta \int_{0}^{t}\|\varphi(\tau)\|_{1}^{2} d \tau .
\end{aligned}
$$

Therefore, substituting the estimates (3.30)-(3.38) in (3.29), and taking $\epsilon$ and $\varepsilon_{4}$ suitably small, we arrive at the desired estimate (3.22). 
For the sake of the closure of the a priori estimate, we need to derive the estimate for $\psi_{x x}$, which is stated in the following lemma.

Lemma 3.6. There exist positive constants $\varepsilon_{5}\left(\leq \varepsilon_{4}\right)$ and $C$ such that if $N(T)+\delta<\varepsilon_{5}$, then it holds that

$$
\left\|\psi_{x}(t)\right\|^{2}+\int_{0}^{t}\left\|\psi_{x x}(\tau)\right\|^{2} d \tau \leq C\left\{\left\|\left(\varphi_{0}, \psi_{0}\right)\right\|_{1}^{2}+\left\|\chi_{0}\right\|^{2}+(N(T)+\delta) \int_{0}^{t} M^{2}(\tau) d \tau\right\}
$$

for an arbitrary $t \in[0, T]$.

Proof. Multiplying $(3.2 \mathrm{~b})$ by $-\psi_{x x} / \rho$ yields

$$
\frac{1}{2}\left(\psi_{x}^{2}\right)_{t}-\left(\psi_{t} \psi_{x}\right)_{x}-u \psi_{x} \psi_{x x}-\frac{p^{\prime}(\rho)}{\rho} \varphi_{x} \psi_{x x}+\mu \frac{\psi_{x x}^{2}}{\rho}=-\frac{g \psi_{x x}}{\rho}-\psi_{x x} \chi-\frac{\tilde{E} \varphi \psi_{x x}}{\rho} .
$$

Here we have used the identity

$$
\psi_{t} \psi_{x x}=\left(\psi_{t} \psi_{x}\right)_{x}-\psi_{t x} \psi_{x}=\left(\psi_{t} \psi_{x}\right)_{x}-\frac{1}{2}\left(\psi_{x}^{2}\right)_{t}
$$

By integrating (3.40) over $[0, t] \times \mathbb{R}_{+}$, we have

$$
\begin{aligned}
& \frac{1}{2}\left\|\psi_{x}(t)\right\|^{2}+\int_{0}^{t}\left(\psi_{t} \psi_{x}\right)(\tau, 0) d \tau+\mu \int_{0}^{t} \int_{0}^{\infty} \frac{\psi_{x x}^{2}}{\rho}(\tau, x) d x d \tau \\
& =\frac{1}{2}\left\|\psi_{0 x}\right\|^{2}+\int_{0}^{t} \int_{0}^{\infty}\left(\left(u \psi_{x}+\frac{p^{\prime}(\rho)}{\rho} \varphi_{x}\right) \psi_{x x}\right)(\tau, x) d x d \tau-\int_{0}^{t} \int_{0}^{\infty} \frac{g \psi_{x x}}{\rho}(\tau, x) d x d \tau \\
& \quad-\int_{0}^{t} \int_{0}^{\infty}\left(\chi \psi_{x x}\right)(\tau, x) d x d \tau-\int_{0}^{t} \int_{0}^{\infty} \frac{\tilde{E} \varphi \psi_{x x}}{\rho}(\tau, x) d x d \tau .
\end{aligned}
$$

We estimate each term in (3.42). Apply integration by parts and (3.4b) to get that

$$
\int_{0}^{t}\left(\psi_{t} \psi_{x}\right)(\tau, 0) d \tau=\psi_{x}(\tau, 0) \psi(\tau, 0)-\int_{0}^{t} \psi(\tau, 0)\left(\psi_{x}(\tau, 0)\right)_{\tau} d \tau=0
$$

Due to (3.11), the third term on the left hand side of (3.42) has the lower bound estimate

$$
\mu \int_{0}^{t} \int_{0}^{\infty} \frac{\psi_{x x}^{2}}{\rho}(\tau, x) d x d \tau \geq c \int_{0}^{t}\left\|\psi_{x x}(\tau)\right\|^{2} d \tau
$$

By using the Schwarz inequality, (1.4), (3.11) and (3.12), the second term on the right hand side of (3.42) is estimated as

$$
\begin{aligned}
& \left|\int_{0}^{t} \int_{0}^{\infty}\left(\left(u \psi_{x}+\frac{p^{\prime}(\rho)}{\rho} \varphi_{x}\right) \psi_{x x}\right)(\tau, x) d x d \tau\right| \\
& \quad \leq \epsilon_{1} \int_{0}^{t}\left\|\psi_{x x}(\tau)\right\|^{2} d \tau+C_{\epsilon_{1}} \int_{0}^{t}\left(\left\|\psi_{x}(\tau)\right\|^{2}+\left\|\varphi_{x}(\tau)\right\|^{2}\right) d \tau
\end{aligned}
$$

where $\epsilon_{1}$ is an arbitrary positive constant and $C_{\epsilon_{1}}$ is a constant depending only on $\epsilon_{1}$. Note that the last integration of (3.45) has been estimated in (3.14) and (3.22). Using (3.13), (1.9), (3.5a) 
and the Schwarz inequality yields

$$
\begin{aligned}
\left|\int_{0}^{t} \int_{0}^{\infty} \frac{g \psi_{x x}}{\rho}(\tau, x) d x d \tau\right| & \leq C \int_{0}^{t} \int_{0}^{\infty}\left(\left|\tilde{u}_{x}\right||(\varphi, \psi)|\left|\psi_{x x}\right|\right)(\tau, x) d x d \tau \\
& \leq C \int_{0}^{t} \int_{0}^{\infty}\left(\left|\tilde{u}_{x}\right|\left(|\varphi|^{2}+|\psi|^{2}+\left|\psi_{x x}\right|^{2}\right)\right)(\tau, x) d x d \tau \\
& \leq C \delta \int_{0}^{t}\left(\left\|\varphi_{x}(\tau)\right\|^{2}+|\varphi(\tau, 0)|^{2}+\left\|\psi_{x}(\tau)\right\|_{1}^{2}\right) d \tau
\end{aligned}
$$

Using integration by parts and (3.4b), it follows that

$$
-\int_{0}^{t} \int_{0}^{\infty}\left(\chi \psi_{x x}\right)(\tau, x) d x d \tau=\int_{0}^{t} \psi_{x}(\tau, 0) \chi(\tau, 0) d \tau+\int_{0}^{t} \int_{0}^{\infty}\left(\chi_{x} \psi_{x}\right)(\tau, x) d x d \tau .
$$

Furthermore, we have

$$
\begin{aligned}
\left|\int_{0}^{t} \psi_{x}(\tau, 0) \chi(\tau, 0) d \tau\right| & \leq \epsilon_{2} \int_{0}^{t}\left|\psi_{x}(\tau, 0)\right|^{2} d \tau+C_{\epsilon_{2}} \int_{0}^{t}|\chi(\tau, 0)|^{2} d \tau \\
& \leq \epsilon_{2} \int_{0}^{t} \int_{0}^{\infty}\left|\left(\psi_{x}^{2}(\tau, x)\right)_{x}\right| d x d \tau+C_{\epsilon_{2}} \int_{0}^{t}|\chi(\tau, 0)|^{2} d \tau \\
& \leq C \epsilon_{2} \int_{0}^{t} \int_{0}^{\infty}\left|\psi_{x}(\tau, x) \| \psi_{x x}(\tau, x)\right| d x d \tau+C_{\epsilon_{2}} \int_{0}^{t}|\chi(\tau, 0)|^{2} d \tau \\
& \leq C \epsilon_{2} \int_{0}^{t}\left(\left\|\psi_{x}(\tau)\right\|^{2}+\left\|\psi_{x x}(\tau)\right\|^{2}\right) d \tau+C_{\epsilon_{2}} \int_{0}^{t}|\chi(\tau, 0)|^{2} d \tau
\end{aligned}
$$

and

$$
\left|\int_{0}^{t} \int_{0}^{\infty}\left(\chi_{x} \psi_{x}\right)(\tau, x) d x d \tau\right| \leq C \int_{0}^{t}\left(\|\varphi(\tau)\|^{2}+\left\|\psi_{x}(\tau)\right\|^{2}\right) d \tau
$$

where $\epsilon_{2}$ is an arbitrary positive constant and $C_{\epsilon_{2}}$ is a constant depending only on $\epsilon_{2}$. Note that the last integrations of (3.48) and (3.49) have been estimated in (3.14) and (3.22). Next, we estimate the last term of (3.42) by (3.11) and (1.9) as

$$
\begin{aligned}
\left|\int_{0}^{t} \int_{0}^{\infty} \frac{\tilde{E} \varphi \psi_{x x}}{\rho}(\tau, x) d x d \tau\right| & \leq C \int_{0}^{t} \int_{0}^{\infty}\left(|\tilde{E}||\varphi|^{2}+|\tilde{E}|\left|\psi_{x x}\right|^{2}\right)(\tau, x) d x d \tau \\
& \leq C \delta \int_{0}^{t}\left(\|\varphi(\tau)\|^{2}+\left\|\psi_{x x}(\tau)\right\|^{2}\right) d \tau
\end{aligned}
$$

At last, substituting the estimates (3.43)-(3.50) in (3.42) and taking $\epsilon_{1}, \epsilon_{2}$ and $\varepsilon_{5}$ suitably small, we arrive at the desired estimate (3.39).

\subsection{Proof of Theorem 1.2}

In this subsection, the proof of Theorem 1.2 is completed.

Proof of Theorem 1.2. Since the lifespan time $T_{0}$ in Lemma 3.1 depends on the Hölder norm of the initial data, we have to show the a priori estimate in the Hölder space for the global solution. Since it is easy to see that $E(t, x)$ is a Hölder continuous function from (1.1a) and (1.1c). 
Thus, exactly as in the proof of the corresponding results in [10], for any $(\varphi, \psi, \chi) \in X(0, T)$, one can obtain the a priori estimate

$$
|\varphi|_{\mathcal{B}_{T}^{1+\frac{\sigma}{2}, 1+\sigma}},|\psi|_{\mathcal{B}_{T}^{1+\frac{\sigma}{2}, 2+\sigma}} \leq C(T)
$$

as long as the Hölder continuity of the function $\xi(t, x):=\int_{0}^{t} E(\tau, x) d \tau$ is verified. In fact, from the equation (1.1c), (1.9) and (3.8), we have

$$
\begin{aligned}
\left|\xi\left(t, x_{1}\right)-\xi\left(t, x_{2}\right)\right| & \leq \int_{0}^{t}\left|E\left(\tau, x_{1}\right)-E\left(\tau, x_{2}\right)\right| d \tau \leq \int_{0}^{t}\left|\int_{x_{1}}^{x_{2}} E_{x}(\tau, x) d x\right| d \tau \\
& \leq\left|x_{1}-x_{2}\right|^{\frac{1}{2}} \int_{0}^{T}|| E_{x}(\tau)|| d \tau=\left|x_{1}-x_{2}\right|^{\frac{1}{2}} \int_{0}^{T}\left\|\left(\rho-\rho_{+}\right)(\tau)\right\| d \tau \\
& \leq\left|x_{1}-x_{2}\right|^{\frac{1}{2}}\left(\int_{0}^{T}\left\|\tilde{\rho}-\rho_{+}\right\| d \tau+\int_{0}^{T} \| \varphi(\tau)|| d \tau\right) \\
& \leq C(T)\left|x_{1}-x_{2}\right|^{\frac{1}{2}}
\end{aligned}
$$

In addition, from (3.12), it follows that

$$
\left|\xi\left(t_{1}, x\right)-\xi\left(t_{2}, x\right)\right| \leq\left|\int_{t_{1}}^{t_{2}}\right| E(\tau, x)|d \tau| \leq C(T)\left|t_{1}-t_{2}\right|
$$

Here $x_{1}, x_{2}, x>0, t_{1}, t_{2}, t \in[0, T]$. The global existence of the solution follows from the standard continuation argument based on the local existence and the a priori estimates in (3.51).

Next we prove the $L^{\infty}$ asymptotic stability mentioned in Theorem 1.2. Since $\|(\varphi, \psi, \chi)(t)\|$ is bounded due to (3.8), it suffices to show that

$$
\left\|\left(\varphi_{x}, \psi_{x}, \chi_{x}\right)(t)\right\| \rightarrow 0, \text { as } t \rightarrow \infty
$$

by virtue of the Sobolev inequality. From (3.2c), (3.2a) and (3.11), it holds that

$$
\begin{aligned}
\left|\frac{d}{d t} \int_{0}^{\infty} \chi_{x}^{2} d x\right| & =2\left|\int_{0}^{\infty} \chi_{x} \chi_{x t} d x\right|=2\left|\int_{0}^{\infty} \varphi \varphi_{t} d x\right| \\
& =2\left|\int_{0}^{\infty} \varphi\left(-u \varphi_{x}-\rho \psi_{x}-\tilde{u}_{x} \varphi-\tilde{\rho}_{x} \psi\right) d x\right| \\
& \leq C \int_{0}^{\infty}\left(|\varphi|\left|\varphi_{x}\right|+|\varphi|\left|\psi_{x}\right|+\left|\tilde{u}_{x}\right||\varphi|^{2}+\left|\tilde{u}_{x}\right||\varphi||\psi|\right) d x
\end{aligned}
$$

Integrating (3.55) over $(0, t)$, then using the Schwarz inequality and (3.5a) yields the estimate

$$
\int_{0}^{t}\left|\frac{d}{d \tau} \int_{0}^{\infty} \chi_{x}^{2}(\tau, x) d x\right| d \tau \leq C \int_{0}^{t} M^{2}(\tau) d \tau .
$$

From Proposition 3.3, the above inequality leads to

$$
\int_{0}^{\infty}\left(\left\|\chi_{x}(\tau)\right\|^{2}+\left|\frac{d}{d \tau}\left\|\chi_{x}(\tau)\right\|^{2}\right|\right) d \tau \leq C
$$

which implies $\left\|\chi_{x}(t)\right\| \rightarrow 0$, as $t \rightarrow \infty$. In a similar way, we can get $\left\|\left(\varphi_{x}, \psi_{x}\right)(t)\right\| \rightarrow 0$, as $t \rightarrow \infty$. Consequently, the proof of Theorem 1.2 is completed. 


\section{Convergence rate and weighted energy estimate}

In this section, we show the convergence rate stated in Theorem 1.3 by using a time and space weighted energy method.

The a priori estimate is obtained in the weighted Sobolev space $X_{\omega}(0, T)$ defined by

$$
X_{\omega}(0, T):=\left\{(\varphi, \psi, \chi) \in X(0, T) \mid(\sqrt{\omega} \varphi, \sqrt{\omega} \psi, \sqrt{\omega} \chi) \in C\left([0, T] ; L^{2}\left(\mathbb{R}_{+}\right)\right)\right\}
$$

We choose the weight function $W(t, x)=\eta(t) \omega(x)$, where $\eta(y)=\omega(y):=(1+y)^{\alpha}$ or $e^{\alpha y}$. For simplicity, the weighted norms $\|\cdot\|_{2, \omega},\|\cdot\|_{\mathrm{a}, \alpha}$ and $\|\cdot\|_{\mathrm{e}, \alpha}$ are defined by

$$
\|f\|_{2, \omega}:=\left\{\int_{0}^{\infty} \omega(x)|f(x)|^{2} d x\right\}^{\frac{1}{2}},\|f\|_{a, \alpha}:=\|f\|_{2,(1+x)^{\alpha}},\|f\|_{e, \alpha}:=\|f\|_{2, e^{\alpha x}} .
$$

We also use the following notation until the end of this paper

$$
\begin{aligned}
L^{2}(t) & :=\eta_{t}(t)\left(\|(\varphi, \psi, \chi)(t)\|_{2, \omega}^{2}+\left\|\left(\varphi_{x}, \psi_{x}\right)(t)\right\|^{2}\right) \\
& +\eta(t)\left(\|\psi(t)\|_{2, \omega_{x x}}^{2}+\|(\varphi, \psi, \chi)(t)\|_{2,\left|\tilde{u}_{x}\right| \omega}^{2}+\|(\varphi, \psi)(t)\|_{2,|\tilde{E}| \omega}^{2}\right) .
\end{aligned}
$$

For the supersonic flow, we summarize the a priori estimate of the solution $\varphi, \psi, \chi$ in $X_{\omega}(0, T)$ which implies Theorem 1.3.

Proposition 4.1. Suppose that the same assumptions as in Theorem 1.3 hold.

(i) (Algebraic decay) Suppose that $(\varphi, \psi, \chi) \in X_{(1+x)^{\alpha}}(0, T)$ is a solution to (3.2) and (3.4) for certain positive constants $\alpha$ and $T$. Then there exist positive constants $\varepsilon_{6}$ and $C$ such that if $N(T)+\delta<\varepsilon_{6}$, then the solution $(\varphi, \psi, \chi)$ satisfies the estimate

$$
\begin{aligned}
& (1+t)^{\alpha+\varepsilon}\left(\|(\varphi, \psi)(t)\|_{1}^{2}+\|\chi(t)\|^{2}\right)+\int_{0}^{t}(1+\tau)^{\alpha+\varepsilon}\left(\|\varphi(\tau)\|_{1}^{2}+\left\|\psi_{x}(\tau)\right\|_{1}^{2}\right) d \tau \\
& \quad+\int_{0}^{t}(1+\tau)^{\alpha+\varepsilon}\left|\left(\varphi, \varphi_{x}, \chi\right)(\tau, 0)\right|^{2} d \tau \leq C\left(\left\|\left(\varphi_{0}, \psi_{0}\right)\right\|_{1}^{2}+\left\|\left(\varphi_{0}, \psi_{0}, \chi_{0}\right)\right\|_{a, \alpha}^{2}\right)(1+t)^{\varepsilon}
\end{aligned}
$$

for arbitrary $t \in[0, T]$ and $\varepsilon>0$.

(ii)(Exponential decay) Suppose that $(\varphi, \psi, \chi) \in X_{e^{\zeta x}}(0, T)$ is a solution to (3.2) and (3.4) for certain positives $\zeta$ and $T$. Then there exist positive constants $\varepsilon_{7}, C, \beta(<\zeta)$ and $\alpha$ satisfying $\alpha \ll \beta$ such that if $N(T)+\delta<\varepsilon_{7}$, then the solution $(\varphi, \psi, \chi)$ satisfies

$$
\begin{aligned}
& e^{\alpha t}\left(\|(\varphi, \psi)(t)\|_{1}^{2}+\|(\varphi, \psi, \chi)(t)\|_{e, \beta}^{2}\right)+\int_{0}^{t} e^{\alpha \tau}\left|\left(\varphi, \varphi_{x}, \chi\right)(\tau, 0)\right|^{2} d \tau \\
& \quad+\int_{0}^{t} e^{\alpha \tau}\left(\|\varphi(\tau)\|_{1}^{2}+\left\|\psi_{x}(\tau)\right\|_{1}^{2}\right) d \tau+\int_{0}^{t} e^{\alpha \tau}\left(\|(\varphi, \psi, \chi)(\tau)\|_{e, \beta}^{2}+\left\|\psi_{x}(\tau)\right\|_{e, \beta}^{2}\right) d \tau \\
& \quad \leq C\left(\left\|\left(\varphi_{0}, \psi_{0}\right)\right\|_{1}^{2}+\left\|\left(\varphi_{0}, \psi_{0}, \chi_{0}\right)\right\|_{e, \beta}^{2}\right) .
\end{aligned}
$$

For the sake of clarity, we divide the proof of Proposition 4.1 into the following three lemmas. 
Lemma 4.2. Suppose that the same assumptions as in Theorem 1.3 hold. Then there exists a positive constant $\varepsilon_{8}$ such that if $N(T)+\delta<\varepsilon_{8}$, it holds that

$$
\begin{aligned}
& \eta(t)\|(\varphi, \psi, \chi)(t)\|_{2, \omega}^{2}+\int_{0}^{t} \eta(\tau)\left(\|(\varphi, \psi, \chi)(\tau)\|_{2, \omega_{x}}^{2}+\left\|\psi_{x}(\tau)\right\|_{2, \omega}^{2}+|\varphi(\tau, 0)|^{2}+|\chi(\tau, 0)|^{2}\right) d \tau \\
& \quad \leq C\left\{\left\|\left(\varphi_{0}, \psi_{0}, \chi_{0}\right)\right\|_{2, \omega}^{2}+\int_{0}^{t} L^{2}(\tau) d \tau\right\}
\end{aligned}
$$

Proof. For convenience, we define the following functionals

$$
\mathcal{E}(\rho, u):=\frac{1}{2}(u-\tilde{u})^{2}+K \tilde{\rho}^{\gamma-1} \Phi\left(\frac{\tilde{\rho}}{\rho}\right), \Phi(s):=s-1-\int_{1}^{s} \eta^{-\gamma} d \eta
$$

Here the energy form $\mathcal{E}$ is equivalent to $|(\varphi, \psi)|^{2}$. That is, there exist two positive constants $c$ and $C$ such that

$$
c\left(\varphi^{2}+\psi^{2}\right) \leq \mathcal{E} \leq C\left(\varphi^{2}+\psi^{2}\right) .
$$

By multiplying (3.2b) by $\psi$, and then using (3.2a) and (3.2c), we find that $\mathcal{E}$ satisfies the equation

$$
\left(\rho \mathcal{E}+\frac{1}{2} \chi^{2}\right)_{t}-\left(G+\mu \psi \psi_{x}\right)_{x}+\mu\left(\psi_{x}\right)^{2}-\frac{1}{2} \tilde{u}_{x} \chi^{2}=R+\tilde{E} \varphi \psi
$$

where

$$
\begin{aligned}
& G:=-\{\rho u \mathcal{E}+[p(\rho)-p(\tilde{\rho})] \psi\}-\frac{1}{2} \tilde{u} \chi^{2} \\
& R:=-\left\{(\rho u-\tilde{\rho} \tilde{u}) \psi+\left[p(\rho)-p(\tilde{\rho})-p^{\prime}(\tilde{\rho}) \varphi\right]\right\} \tilde{u}_{x}-\frac{1}{\tilde{\rho}} \varphi \psi p(\tilde{\rho})_{x} .
\end{aligned}
$$

Multiplying (4.6) by a weight function $W(t, x)$ yields

$$
\begin{aligned}
& \left(W\left(\rho \mathcal{E}+\frac{1}{2} \chi^{2}\right)\right)_{t}-\left(W G+\mu W \psi \psi_{x}-\frac{\mu}{2} W_{x} \psi^{2}\right)_{x}+W_{x} G+\mu W \psi_{x}^{2} \\
& =W_{t}\left(\rho \mathcal{E}+\frac{1}{2} \chi^{2}\right)+\frac{\mu}{2} W_{x x} \psi^{2}+W R+\frac{1}{2} W \tilde{u}_{x} \chi^{2}+W \tilde{E} \varphi \psi
\end{aligned}
$$

One can split $G$ as $G_{1}+G_{2}$ (cf. [18]), where

$$
\begin{aligned}
G_{1} & :=\frac{K \gamma \rho_{+}^{\gamma-2}\left|u_{+}\right|}{2} \varphi^{2}+\frac{\rho_{+}\left|u_{+}\right|}{2} \psi^{2}-K \gamma \rho_{+}^{\gamma-1} \varphi \psi-\frac{1}{2} \tilde{u} \chi^{2} \\
G_{2} & :=-\frac{K \gamma \rho_{+} u_{+}}{2 \rho^{2}}\left(\tilde{\rho}^{\gamma-1}-\rho_{+}^{\gamma-3} \rho^{2}\right) \varphi^{2}-K \rho_{+} u_{+} \tilde{\rho}^{\gamma-1}\left\{\Phi\left(\frac{\tilde{\rho}}{\rho}\right)-\frac{\gamma}{2}\left(\frac{\tilde{\rho}}{\rho}-1\right)^{2}\right\} \\
& -K \gamma\left(\tilde{\rho}^{\gamma-1}-\rho_{+}^{\gamma-1}\right) \varphi \psi-K \tilde{\rho}^{\gamma}\left\{\left(\frac{\rho}{\tilde{\rho}}\right)^{\gamma}-1-\gamma\left(\frac{\rho}{\tilde{\rho}}-1\right)\right\} \psi-\left(\rho u-\rho_{+} u_{+}\right) \mathcal{E} .
\end{aligned}
$$

The condition $M_{+}>1$ and (2.10) guarantee

$$
\begin{aligned}
G_{1} & =\left(\frac{p^{\prime}\left(\rho_{+}\right)^{3 / 2}}{2 \rho_{+}} \varphi^{2}+\frac{\rho_{+} \sqrt{p^{\prime}\left(\rho_{+}\right)}}{2} \psi^{2}\right)\left(M_{+}-1\right)+\frac{\sqrt{p^{\prime}\left(\rho_{+}\right)}}{2 \rho_{+}}\left(\sqrt{p^{\prime}\left(\rho_{+}\right)} \varphi-\rho_{+} \psi\right)^{2}-\frac{1}{2} \tilde{u} \chi^{2} \\
& \geq c\left(\varphi^{2}+\psi^{2}+\chi^{2}\right) .
\end{aligned}
$$


From (1.9), (4.5) and the inequalities $\left|\Phi(s)-\frac{\gamma}{2}(s-1)^{2}\right| \leq C|s-1|^{3},\left|s^{\gamma}-1-\gamma(s-1)\right| \leq C|s-1|^{2}$ for $|s-1| \ll 1$, we have

$$
\left|G_{2}\right| \leq C(N(t)+\delta)\left(\varphi^{2}+\psi^{2}\right)
$$

Integrating (4.8) over $[0, t] \times \mathbb{R}_{+}$and noticing the boundary conditions (1.3a), (1.6) and (3.4b) yields

$$
\begin{aligned}
& \int_{0}^{\infty}\left(W\left(\rho \mathcal{E}+\frac{1}{2} \chi^{2}\right)\right)(t, x) d x+\int_{0}^{t} \eta(\tau)\left|u_{b}\right|\left(\rho(\tau, 0) \mathcal{E}(\tau, 0)+|\chi(\tau, 0)|^{2}\right) d \tau \\
& +\int_{0}^{t} \int_{0}^{\infty}\left(W_{x} G_{1}\right)(\tau, x) d x d \tau+\int_{0}^{t} \int_{0}^{\infty}\left(\mu W \psi_{x}^{2}\right)(\tau, x) d x d \tau \\
& =\int_{0}^{\infty}\left(W\left(\rho \mathcal{E}+\frac{1}{2} \chi^{2}\right)\right)(0, x) d x+\int_{0}^{t} \int_{0}^{\infty}\left(W_{t}\left(\rho \mathcal{E}+\frac{1}{2} \chi^{2}\right)\right)(\tau, x) d x d \tau \\
& +\int_{0}^{t} \int_{0}^{\infty}\left(\frac{\mu}{2} W_{x x} \psi^{2}\right)(\tau, x) d x d \tau+\int_{0}^{t} \int_{0}^{\infty}(W R)(\tau, x) d x d \tau \\
& \quad-\int_{0}^{t} \int_{0}^{\infty}\left(W_{x} G_{2}\right)(\tau, x) d x d \tau+\int_{0}^{t} \int_{0}^{\infty}\left(\frac{1}{2} W \tilde{u}_{x} \chi^{2}\right)(\tau, x) d x d \tau \\
& \quad+\int_{0}^{t} \int_{0}^{\infty}(W \tilde{E} \varphi \psi)(\tau, x) d x d \tau
\end{aligned}
$$

Owing to the Schwarz inequality, it follows that

$$
\left|\int_{0}^{t} \int_{0}^{\infty}(W \tilde{E} \varphi \psi)(\tau, x) d x d \tau\right| \leq C \int_{0}^{t} \int_{0}^{\infty}\left(W \tilde{E}\left(|\varphi|^{2}+|\psi|^{2}\right)\right)(\tau, x) d x d \tau
$$

Therefore, substituting (4.10), (4.11) and (4.13) in (4.12), and then taking $N(T)+\delta$ suitably small, we prove the desired estimate (4.3).

Lemma 4.3. There exists a positive constant $\varepsilon_{9}\left(\leq \varepsilon_{8}\right)$ such that if $N(T)+\delta<\varepsilon_{9}$, then

$$
\begin{gathered}
\eta(t)\left\|\varphi_{x}(t)\right\|^{2}+\int_{0}^{t} \eta(\tau)\left(\|\varphi(\tau)\|_{1}^{2}+\left|\varphi_{x}(\tau, 0)\right|^{2}\right) d \tau \leq C\left(\left\|\varphi_{0 x}\right\|^{2}+\left\|\left(\varphi_{0}, \psi_{0}, \chi_{0}\right)\right\|_{2, \omega}^{2}\right) \\
+C \int_{0}^{t} L^{2}(\tau) d \tau+C(N(t)+\delta) \int_{0}^{t} \eta(\tau) M^{2}(\tau) d \tau .
\end{gathered}
$$

Proof. Multiplying (3.28) by a weight function $\eta=\eta(t)$, and then integrating it over $[0, t] \times \mathbb{R}_{+}$, we can obtain the desired estimate (4.14) by the similar computation as in the proof of Lemma 3.5 .

Lemma 4.4. There exists a positive constant $\varepsilon_{10}\left(\leq \varepsilon_{9}\right)$ such that if $N(T)+\delta<\varepsilon_{10}$, then

$$
\begin{gathered}
\eta(t)\left\|\psi_{x}(t)\right\|^{2}+\int_{0}^{t} \eta(\tau)\left\|\psi_{x x}(\tau)\right\|^{2} d \tau \leq C\left(\left\|\left(\varphi_{0 x}, \psi_{0 x}\right)\right\|^{2}+\left\|\left(\varphi_{0}, \psi_{0}, \chi_{0}\right)\right\|_{2, \omega}^{2}\right) \\
+C \int_{0}^{t} L^{2}(\tau) d \tau+C(N(t)+\delta) \int_{0}^{t} \eta(\tau) M^{2}(\tau) d \tau .
\end{gathered}
$$

Proof. Multiplying (3.40) by a weight function $\eta=\eta(t)$, and then integrating it over $[0, t] \times \mathbb{R}_{+}$, we can obtain the desired estimate (4.15) by the similar computation as in the proof of Lemma 3.6 . 
Next we prove Proposition 4.1.

Proof of Proposition 4.1. Summing up the estimates (4.3), (4.14) and (4.15) and taking $N(T)+\delta$ suitably small, we have

$$
\begin{aligned}
& \eta(t)\left(\left\|\left(\varphi_{x}, \psi_{x}\right)\right\|^{2}+\|(\varphi, \psi, \chi)\|_{2, \omega}^{2}\right)+\int_{0}^{t} \eta(\tau)\left|\left(\varphi, \varphi_{x}, \chi\right)(\tau, 0)\right|^{2} d \tau \\
& \quad+\int_{0}^{t} \eta(\tau)\left(\|(\varphi, \psi, \chi)(\tau)\|_{2, \omega_{x}}^{2}+\|\varphi(\tau)\|_{1}^{2}+\left\|\psi_{x}(\tau)\right\|_{2, \omega}^{2}+\left\|\psi_{x x}(\tau)\right\|^{2}\right) d \tau \\
& \leq C\left(\left\|\left(\varphi_{0 x}, \psi_{0 x}\right)\right\|^{2}+\left\|\left(\varphi_{0}, \psi_{0}, \chi_{0}\right)\right\|_{2, \omega}^{2}\right)+C \int_{0}^{t} L^{2}(\tau) d \tau
\end{aligned}
$$

Substituting $\omega(x)=(1+x)^{\beta}$ and $\chi(t)=(1+t)^{\xi}$ in (4.16) for $\beta \in[0, \alpha]$ and $\xi \geq 0$, and using the Poincaré type inequality (3.5) gives

$$
\begin{aligned}
& (1+t)^{\xi}\left(\left\|\left(\varphi_{x}, \psi_{x}\right)(t)\right\|^{2}+\|(\varphi, \psi, \chi)(t)\|_{a, \beta}^{2}\right)+\int_{0}^{t}(1+\tau)^{\xi}\left|\left(\varphi, \varphi_{x}, \chi\right)(\tau, 0)\right|^{2} d \tau \\
& \quad+\int_{0}^{t}(1+\tau)^{\xi}\left(\beta\|(\varphi, \psi, \chi)(\tau)\|_{a, \beta-1}^{2}+\left\|\psi_{x}(\tau)\right\|_{a, \beta}^{2}+\|\varphi(\tau)\|_{1}^{2}+\left\|\psi_{x x}(\tau)\right\|^{2}\right) d \tau \\
& \leq C\left(\left\|\left(\varphi_{0}, \psi_{0}\right)\right\|_{1}^{2}+\left\|\left(\varphi_{0}, \psi_{0}, \chi_{0}\right)\right\|_{a, \beta}^{2}\right)+C \beta(\beta-1) \int_{0}^{t}(1+\tau)^{\xi}\|\psi(\tau)\|_{a, \beta-2}^{2} d \tau \\
& \quad+C \xi \int_{0}^{t}(1+\tau)^{\xi-1}\left(\|(\varphi, \psi, \chi)(\tau)\|_{a, \beta}^{2}+\left\|\left(\varphi_{x}, \psi_{x}\right)(\tau)\right\|^{2}\right) d \tau .
\end{aligned}
$$

Applying the induction argument to (4.17) in $\beta$ and $\xi$, it holds that for $\xi=0,1, \ldots,[\alpha]$

$$
\begin{aligned}
& (1+t)^{\xi}\left(\left\|\left(\varphi_{x}, \psi_{x}\right)(t)\right\|^{2}+\|(\varphi, \psi, \chi)(t)\|_{a, \alpha-\xi}^{2}\right)+\int_{0}^{t}(1+\tau)^{\xi}\left|\left(\varphi, \varphi_{x}, \chi\right)(\tau, 0)\right|^{2} d \tau \\
& \quad+\int_{0}^{t}(1+\tau)^{\xi}\left((\alpha-\xi)\|(\varphi, \psi, \chi)(\tau)\|_{a, \alpha-\xi-1}^{2}+\left\|\psi_{x}(\tau)\right\|_{a, \alpha-\xi}^{2}+\|\varphi(\tau)\|_{1}^{2}+\left\|\psi_{x x}(\tau)\right\|^{2}\right) d \tau \\
& \leq C\left(\left\|\left(\varphi_{0}, \psi_{0}\right)\right\|_{1}^{2}+\left\|\left(\varphi_{0}, \psi_{0}, \chi_{0}\right)\right\|_{a, \alpha}^{2}\right) .
\end{aligned}
$$

In fact, letting $\beta=\alpha$ and $\xi=0$ in (4.17). For $\alpha<1$, we have (4.18) with $\xi=0$. Next we take $1 \leq \alpha<2$. Letting $\beta=\alpha-1$ and $\xi=1$ and using (4.18) with $\xi=0$, we have the estimate (4.18) with $\xi=1$. Repeating the same procedure yields the desired estimate (4.18) for any $\alpha \geq 0$. Therefore, if $\alpha$ is an integer, then

$$
\begin{aligned}
& (1+t)^{\alpha}\left(\|(\varphi, \psi)(t)\|_{1}^{2}+\|\chi(t)\|^{2}\right)+\int_{0}^{t}(1+\tau)^{\alpha}\left(\left\|\psi_{x}(\tau)\right\|_{1}^{2}+\|\varphi(\tau)\|_{1}^{2}\right) d \tau \\
& \quad+\int_{0}^{t}(1+\tau)^{\alpha}\left|\left(\varphi, \varphi_{x}, \chi\right)(\tau, 0)\right|^{2} d \tau \leq C\left(\left\|\left(\varphi_{0}, \psi_{0}\right)\right\|_{1}^{2}+\left\|\left(\varphi_{0}, \psi_{0}, \chi_{0}\right)\right\|_{a, \alpha}^{2}\right) .
\end{aligned}
$$

If $\alpha$ is non-integer, from the Hölder inequality, we can arrive at the desired estimate (4.1). Since this proof is similar to [14], we omit the details.

Next, we prove the estimate (4.2). Substituting $\omega(x)=e^{\beta x}$ and $\chi(t)=e^{\alpha t}$ in (4.16) for 
$\beta<\zeta$, and using the Poincaré type inequality (3.5) yields

$$
\begin{aligned}
& e^{\alpha t}\left(\|(\varphi, \psi, \chi)(t)\|_{e, \beta}^{2}+\left\|\left(\varphi_{x}, \psi_{x}\right)(t)\right\|^{2}\right)+\int_{0}^{t} e^{\alpha \tau}\left|\left(\varphi, \varphi_{x}, \chi\right)(\tau, 0)\right|^{2} d \tau \\
& +\int_{0}^{t} e^{\alpha \tau}\left(\beta\|(\varphi, \psi, \chi)(\tau)\|_{e, \beta}^{2}+\|\varphi(\tau)\|_{1}^{2}+\left\|\psi_{x x}(\tau)\right\|^{2}+\left\|\psi_{x}(\tau)\right\|_{e, \beta}^{2}\right) d \tau \\
& \leq C\left(\left\|\left(\varphi_{0}, \psi_{0}, \chi_{0}\right)\right\|_{e, \beta}^{2}+\left\|\left(\varphi_{0 x}, \psi_{0 x}\right)\right\|^{2}\right)+C \alpha \int_{0}^{t} e^{\alpha \tau}\left\|\left(\varphi_{x}, \psi_{x}\right)(\tau)\right\|^{2} d \tau \\
& +C \delta \int_{0}^{t} e^{\alpha \tau}\left(|(\varphi, \chi)(\tau, 0)|^{2}+\|\varphi(\tau)\|^{2}+\left\|\left(\varphi_{x}, \psi_{x}\right)(\tau)\right\|^{2}\right) d \tau \\
& +C\left(\alpha+\beta^{2}\right) \int_{0}^{t} e^{\alpha \tau}\|(\varphi, \psi, \chi)(\tau)\|_{e, \beta}^{2} d \tau .
\end{aligned}
$$

Thus, taking $\delta, \beta$ and $\alpha$ suitably small, we arrive at the desired a priori estimate (4.2).

\section{Acknowledgements}

Zhang's work is partially supported by the National Science Foundation of China (No.201113115) and the Fundamental Research Funds for the Central Universities (No.111065201).

\section{References}

[1] D. Chae, On the nonexistence of global weak solutions to the Navier-Stokes-Poisson equations in $R^{N}$, Comm. Partial Differential Equations, 35 (2010), 535-557.

[2] P. Degond, Mathematical modelling of microelectronics semiconductor devices//Some Current Topics on Nonlinear Conservation Laws, AMS/IP Stud Adv Math, 15. Providence, RI: Amer Math Soc, 266 (2000), 77-110.

[3] D. Donatelli, Local and global existence for the coupled Navier-Stokes-Poisson problem, Quart.Appl. Math., 61 (2003), 345-361.

[4] D. Donatelli and P. Marcati, A quasineutral type limit for the Navier-Stokes-Poisson system with large data, Nonlinearity, 21 (2008), 135-148.

[5] R.-J. Duan and X.-F. Yang, Stability of rarefaction wave and boundary layer for outflow problem on the two-fluid Navier-Stokes-Poisson equations, Commun. Pure Appl. Anal., 12 (2013), 985-1014.

[6] C.-C. Hao and H.-L. Li, Global existence for compressible Navier-Stokes-Poisson equations in three and higher dimensions, J. Differential Equations, 246 (2009), 4791-4812.

[7] L.Hsiao and H.-L. Li, Compressible Navier-Stokes-Poisson equations, Acta Math Sci-B, 30 (2010), 1937-1948. 
[8] F.-M. Huang and X.-H. Qin, Stability of boundary layer and rarefaction wave to an outflow problem for compressible Navier-Stokes equations under large perturbation, J. Differential Equations, 246 (2009), 4077-4096.

[9] Y. Kagei and S. Kawashima, Stability of planar stationary solutions to the compressible Navier-Stokes equation on the half space, Comm. Math. Phys., 266 (2006), 401-430.

[10] S. Kawashima, S. Nishibata and P.-C. Zhu, Asymptotic stability of the stationary solution to the compressible Navier-Stokes equations in the half space, Comm. Math.Phys., 240 (2003), 483-500.

[11] S. Kawashima and P.-C. Zhu, Asymptotic stability of nonlinear wave for the compressible Navier-Stokes equations in the half space, J. Differential Equations, 244 (2008), 3151-3179.

[12] H.-L. Li, T. Yang and C. Zou, Time asymptotic behavior of the bipolar Navier-StokesPoisson system, Acta Math. Sci.-B, 29 (2009), 1721-1736.

[13] H.-L. Li, A. Matsumura and G.-J. Zhang, Optimal decay rate of the compressible NavierStokes-Poisson system in $R^{3}$, Arch. Ration. Mech. Anal., 196 (2010), 681-713.

[14] A. Matsumura and K. Nishihara, Asymptotic stability of traveling waves for scalar viscous conservation laws with non-convex nonlinearity, Comm. Math.Phys., 165 (1994), 83-96.

[15] A. Matsumura and M. Mei, Convergence to travelling fronts of solutions of the p-system with viscosity in the presence of a boundary, Arch. Ration. Mech. Anal., 146 (1999), 1-22.

[16] A. Matsumura and K. Nishihara, Asymptotic toward the rarefaction waves for solutions of viscous p-system with boundary effect, Quart. Appl. Math., 58 (2000), 69-83.

[17] A. Matsumura and K. Nishihara, Large-time behavior of solutions to an inflow problem in the half space for a one-dimensional system of compressible viscous gas, Comm. Math. Phys., 222 (2001), 449-474.

[18] T. Nakamura, S. Nishibata and T. Yuge, Convergence rate of solutions toward stationary solutions to the compressible Navier-Stokes equation in a half line, J.Differential Equations, 241 (2007), 94-111.

[19] T. Nakamura and S. Nishibata, Convergence rate toward planar stationary waves for compressible viscous fluid in multidimensional half space, SIAM J. Math. Anal., 41 (2009), 1757-1791.

[20] K. Nishihara, T. Yang and H.-J. Zhao, Nonlinear stablility of strong rarefaction waves for compressible Navier-Stokes equations, SIAM J. Math. Anal., 35 (2004), 1561-1597. 
[21] Z. Tan, T. Yang, H.-J. Zhao and Q.-Y. Zou, Global solutions to the one-dimensional compressible Navier-Stokes-Poisson equations with large data, SIAM J. Math. Anal., 45 (2013), 547-571.

[22] S. Wang and S. Jiang, The convergence of the Navier-Stokes-Poisson system to the incompressible Euler equations, Comm. Partial Differential Equations, 31 (2006), 571-591.

[23] W.-K. Wang and Z.-G. Wu, Pointwise estimates of solution for the Navier-Stokes-Poisson equations in multi-dimensions, J. Differential Equations, 248 (2010), 1617-1636.

[24] S. Wiggins, Introduction to Applied Nonlinear Dynamical Systems and Chaos, SpringerVerlag Press, 2nd edition, New York, 2003.

[25] G.-J. Zhang, H.-L. Li and C.-J. Zhu, Optimal decay rate of the non-isentropic compressible Navier-Stokes-Poisson system in $R^{3}$, J. Differential Equations, 250 (2011), 866-891.

[26] F. Zhou and Y.-P. Li, Convergence rate of solutions toward stationary solutions to the bipolar Navier-Stokes-Poisson equations in a half line, Bound. Value Probl., 124 (2013), 22 pp. 